Technische

Universität

Berlin

Kai Volgmann, Viktor Epp, Julia Langer, Bernhard Stanje, Jessica Heine, Suliman Nakhal, Martin Lerch, Martin Wilkening, Paul Heitjans

\title{
Solid-State NMR to Study Translational Li Ion Dynamics in Solids with Low-Dimensional Diffusion Pathways
}

Open Access via institutional repository of Technische Universität Berlin

Document type

Journal article | Published version

(i. e. publisher-created published version, that has been (peer-) reviewed and copyedited; also known as:

Version of Record (VOR), Final Published Version)

This version is available at

https://doi.org/10.14279/depositonce-12529

Citation details

Volgmann, K., Epp, V., Langer, J., Stanje, B., Heine, J., Nakhal, S., Lerch, M., Wilkening, M., \& Heitjans, P. (2017). Solid-State NMR to Study Translational Li lon Dynamics in Solids with Low-Dimensional Diffusion Pathways. In Zeitschrift für Physikalische Chemie (Vol. 231, Issues 7-8, pp. 1215-1241). Walter de Gruyter GmbH. https://doi.org/10.1515/zpch-2017-0952

Terms of use

This work is protected by copyright and/or related rights. You are free to use this work in any way permitted by the copyright and related rights legislation that applies to your usage. For other uses, you must obtain permission from the rights-holder(s). 
Kai Volgmann, Viktor Epp, Julia Langer, Bernhard Stanje, Jessica Heine, Suliman Nakhal, Martin Lerch, Martin Wilkening* and Paul Heitjans*

\section{Solid-State NMR to Study Translational Li lon Dynamics in Solids with Low- Dimensional Diffusion Pathways}

DOI 10.1515/zpch-2017-0952

Received March 1, 2017; accepted June 1, 2017

Abstract: Fundamental research on lithium ion dynamics in solids is important to develop functional materials for, e.g. sensors or energy storage systems. In many cases a comprehensive understanding is only possible if experimental data are compared with predictions from diffusion models. Nuclear magnetic resonance (NMR), besides other techniques such as mass tracer or conductivity measurements, is known as a versatile tool to investigate ion dynamics. Among the various time-domain NMR techniques, NMR relaxometry, in particular, serves not only to measure diffusion parameters, such as jump rates and activation energies, it is also useful to collect information on the dimensionality of the underlying diffusion process. The latter is possible if both the temperature and, even more

*Corresponding authors: Martin Wilkening, Institute of Physical Chemistry and Electrochemistry, Leibniz Universität Hannover, Callinstr. 3 - 3a, D-30167 Hannover, Germany; and Institute of Chemistry and Technology of Materials, Christian Doppler Laboratory for Lithium Batteries, Graz University of Technology (NAWI Graz), Stremayrgasse 9, A-8010 Graz, Austria, e-mail: wilkening@tugraz.at; and Paul Heitjans, Institute of Physical Chemistry and Electrochemistry, Leibniz Universität Hannover, Callinstr. 3 - 3a, D-30167 Hannover, Germany; and ZFM - Center for Solid State Chemistry and New Materials, Leibniz Universität Hannover, Callinstr. 3 - 3a, D-30167 Hannover, Germany, e-mail: heitjans@pci.uni-hannover.de Kai Volgmann and Jessica Heine: Institute of Physical Chemistry and Electrochemistry, Leibniz Universität Hannover, Callinstr. 3 - 3a, D-30167 Hannover, Germany; and ZFM - Center for Solid State Chemistry and New Materials, Leibniz Universität Hannover, Callinstr. 3 - 3a, D-30167 Hannover, Germany

Viktor Epp: Institute of Physical Chemistry and Electrochemistry, Leibniz Universität Hannover, Callinstr. 3 - 3a, D-30167 Hannover, Germany; and Institute of Chemistry and Technology of Materials, Christian Doppler Laboratory for Lithium Batteries, Graz University of Technology (NAWI Graz), Stremayrgasse 9, A-8010 Graz, Austria

Julia Langer and Bernhard Stanje: Institute of Chemistry and Technology of Materials, Christian Doppler Laboratory for Lithium Batteries, Graz University of Technology (NAWI Graz), Stremayrgasse 9, A-8010 Graz, Austria

Suliman Nakhal and Martin Lerch: Institut für Chemie, Sekr. C2, Technische Universität Berlin, Straße des 17. Juni 135, D-10623 Berlin, Germany 
important, the frequency dependence of the diffusion-induced relaxation rates of actually polycrystalline materials is analyzed. Here we present some recent systematic relaxometry case studies using model systems that exhibit spatially restricted $\mathrm{Li}$ ion diffusion. Whenever possible we compare our results with data from other techniques as well as current relaxation models developed for 2D and 1D diffusion. As an example, 2D ionic motion has been verified for the hexagonal form of $\mathrm{LiBH}_{4}$; in the high-temperature limit the diffusion-induced ${ }^{7} \mathrm{Li}$ NMR spinlattice relaxation rates follow a logarithmic frequency dependence as is expected from models introduced for 2D diffusion. A similar behavior has been found for $\mathrm{Li}_{x} \mathrm{NbS}_{2}$. In $\mathrm{Li}_{12} \mathrm{Si}_{7}$ a quasi-1D diffusion process seems to be present that is characterized by a square root frequency dependence and a temperature behavior of the ${ }^{7} \mathrm{Li}$ NMR spin-lattice relaxation rates as predicted. Most likely, parts of the Li ions diffuse along the $\mathrm{Si}_{5}$ rings that form chains in the Zintl phase.

Keywords: diffusion; dimensionality; lithium; solid-state NMR; spin-lattice relaxation.

\section{Introduction}

Fundamental research on lithium diffusion in solids using nuclear magnetic resonance (NMR) has been carried out since the 1950s. Very first examples include the study of Li self-diffusion in lithium metal by temperature-dependent NMR spin-lattice relaxation (SLR) [1, 2]. Until today quite many NMR methods have been proven as versatile tools to study $\mathrm{Li}$ ion dynamics in numerous compounds such as crystalline inorganic materials, glasses, polymers and so forth [3, 4]. NMR offers a powerful collection of techniques with which dynamics in solids and liquids can be probed over a large times scale. The majority of the studies published over the last decades are concerned with the measurements of jump rates and activation energies as well as the elucidation of geometric aspects of translational and rotational dynamics. Time-domain SLR NMR is the method of choice to study Li ion self-diffusion processes if information on jump rates and activation energies are needed [5-9]. With NMR relaxometry it is possible to probe both local hopping processes as well as long-range ion transport $[7,10]$.

Most interestingly, in favorite cases NMR is also able to distinguish between diffusion processes characterized by their dimensionality [11, 12]. For that purpose powder samples are sufficient and there is no need to investigate single crystals. This information is included in both the temperature and frequency dependence of the corresponding diffusion-induced ${ }^{6} \mathrm{Li}$ and ${ }^{7} \mathrm{Li}$ NMR SLR rates. As yet, only a few studies took up the challenge to use NMR relaxometry to describe 
low-dimensional translational Li ion dynamics in crystalline materials [10, 12, 13]. If ionic jump diffusion is utterly restricted to two dimensions or even to only one dimension, the diffusion-induced relaxation rates are expected to show a characteristic temperature and frequency dependence being an immediate consequence of the constraints of the lattice.

The deviations expected for 2D and 1D are referred to the so-called standard behavior that applies to isotropic, i.e. 3D, uncorrelated jump diffusion. As early as 1948 Bloembergen, Purcell and Pound (BPP) described this type of motion with a single exponential correlation function $G(t)$, which characterizes the temporal evolution of the internal fluctuating dipolar magnetic fields the diffusing ions are subjected to [14]. The Fourier transform of $G(t)$ leads to the spectral density function $J(\omega)$; the spin-lattice relaxation rate $T_{1}^{-1}$, being the primary observable in NMR relaxometry if carried out in the laboratory frame of reference, is directly proportional to $J\left(\omega_{0}\right) . \omega_{0}$ denotes the angular resonance frequency, the Larmor frequency, of the nucleus under investigation. Assuming Arrhenius behavior for the correlation rate $\tau_{\mathrm{c}}^{-1}$ characterizing the decay of $G(t)$, the ideal BPP-type response leads to a symmetric relaxation rate peak if $T_{1}^{-1}$ is plotted on a logarithmic scale vs. the inverse temperature. While at the peak maximum the correlation rate can be estimated according to $\omega_{0} \tau_{c} \approx 1$, usually $\tau_{c}$ is very similar to the mean jump rate $\tau$, the flanks contain information on (i) motional correlation effects and (ii) the dimensionality of the diffusion process. Correlations of successive jumps, e.g. due to Coulomb interactions or structural disorder [15], cause deviations from the quadratic frequency dependence of $T_{1}^{-1}$ in the low-temperature regime, i.e. in the regime characterized by $\omega_{0} \tau_{c} \gg 1$. If, on the other hand, we have to deal with $2 \mathrm{D}$ or $1 \mathrm{D}$ diffusion a characteristic asymmetry of the SLR NMR rate peak is predicted by the (semi-empirical) relaxation models introduced by Richards (2D) [16] and Sholl (1D) [17]. Most importantly, in the high temperature limit of the peak $\left(\omega_{0} \tau_{c} \ll 1\right)$ the rates should reveal characteristic dependencies on $\omega_{0}$. This behavior is in stark contrast to 3D diffusion for which no such dependence should show up. While for $1 \mathrm{D}$ diffusion the relaxation models predict a square root dependence, a logarithmic one should govern jump diffusion being confined to two dimensions; see the

Tab. 1: Limiting behavior of $T_{1}^{-1}\left(\omega_{0} \tau_{c}\right)$ in the regime $\omega_{0} \tau_{c} \ll 1$, i.e. for the high-temperature flank of the diffusion-induced rate peak; note that for uncorrelated diffusion $T_{1}^{-1}$ is proportional to $\omega_{0}^{-2}$ in the regime $\omega_{0} \tau_{c} \gg 1$ independent of the dimensionality of the diffusion process.

\begin{tabular}{ll}
\hline & $\omega_{0} \tau_{c} \ll 1$ \\
\hline $3 D$ & $\propto \tau_{c}$ \\
2D & $\propto \tau_{c} \ln \left(1 / \omega_{0} \tau_{c}\right)$ \\
1D & $\propto \tau_{c}\left(\omega_{0} \tau_{c}\right)^{-0.5}$ \\
\hline
\end{tabular}


summary in Table 1, adapted from Ref. [5]. The latter case is expected to be met in, e.g. layered materials with the Li ions located between the layers. In principle, the same holds true for spin-lock SLR NMR performed in the rotating frame of reference (spin-locking angular frequency $\omega_{1} \ll \omega_{0}$ ) whereby the diffusion-induced peak is shifted to lower temperatures. Up to now, only few examples have, however, been presented that clearly support these predictions by theory $[11,18]$.

Worth noting, the frequency dependence of the high- $T$ flank of the $T_{1}^{-1}$ rates is often difficult to access experimentally. Then, it can be supported by field-cycling (FC) NMR measurements of SLR rates. FC NMR enables direct access to $T_{1}^{-1}(\omega)$ over a wide frequency range, see Ref. [19], and may help us to further characterize lowdimensional jump diffusion.

In the following we will present a short overview of recently studied compounds in our labs. Whereas earlier investigations put emphasis on 2D diffusion in $\mathrm{Li}_{x} \mathrm{TiS}_{2}$ and the quasi $1 \mathrm{D}$ diffusion in $\beta$-eucryptite $\left(\mathrm{LiAlSiO}_{4}\right)$, for example see Refs. [7, 11, 20-27], the present collection comprises, on the one hand, layer structured materials such as hexagonal $\mathrm{LiBH}_{4}[12,28], \mathrm{Li}_{x} \mathrm{NbS}_{2}[29,30], \mathrm{Li}_{0.17} \mathrm{SnS}_{2}[31]$, $\mathrm{LiC}_{6}$ [32] and, in a companion article, $\mathrm{Li}_{2} \mathrm{Ti}_{3} \mathrm{O}_{7}$ (as well as isostructured $\mathrm{Na}_{2} \mathrm{Ti}_{3} \mathrm{O}_{7}$ ) [13]. On the other hand some silicides, sulfides and oxides with channels are treated. While for $\mathrm{Li}_{0.7} \mathrm{Nb}_{3} \mathrm{~S}_{4}, \mathrm{Li}_{x} \mathrm{Na}_{2-x} \mathrm{Ti}_{6} \mathrm{O}_{13}$ [33], $\mathrm{LiBi}_{3} \mathrm{~S}_{5}$ [34] and ramsdellite-type $\mathrm{Li}_{2} \mathrm{Ti}_{3} \mathrm{O}_{7}$ [35-37] the ions are expected to move inside the channels, the stacked $\mathrm{Si}_{5}$ rings in $\mathrm{Li}_{12} \mathrm{Si}_{7}$ [38-40] guide the ions outside the channels to follow quasi 1D diffusion.

\section{Li-ion conductors with two-dimensional diffusion pathways}

\section{$2.1 \mathrm{LiBH}_{4}$}

The hexagonal modification of lithium boron hydride [41-43] turned out to be a suitable model system to study 2D ionic diffusion $[12,28]$. Below the phase transition temperature, $T_{\mathrm{p}}$, of $380 \mathrm{~K}$ lithium boron hydride crystallizes with orthorhombic symmetry (Pnma). The crystal structure of the orthorhombic modification allows for 3D diffusion. Translational Li ion diffusion is, however, much slower than the rotational dynamics of the $\mathrm{BH}_{4}$ anion unit. Thus, the ${ }^{6} \mathrm{Li}$ and ${ }^{7} \mathrm{Li} \mathrm{NMR} \mathrm{SLR}$ rates are completely governed by the dynamics of their surroundings [12]. This great difference in Li ion diffusivity is best seen if the corresponding ${ }^{7} \mathrm{Li} \mathrm{NMR}$ and ${ }^{6} \mathrm{Li}$ NMR spectra are regarded $[12,28]$. Whereas in orthorhombic $\mathrm{LiBH}_{4}$, because of poor Li exchange, the lines are dipolarly broadened, sharp NMR lines show up 

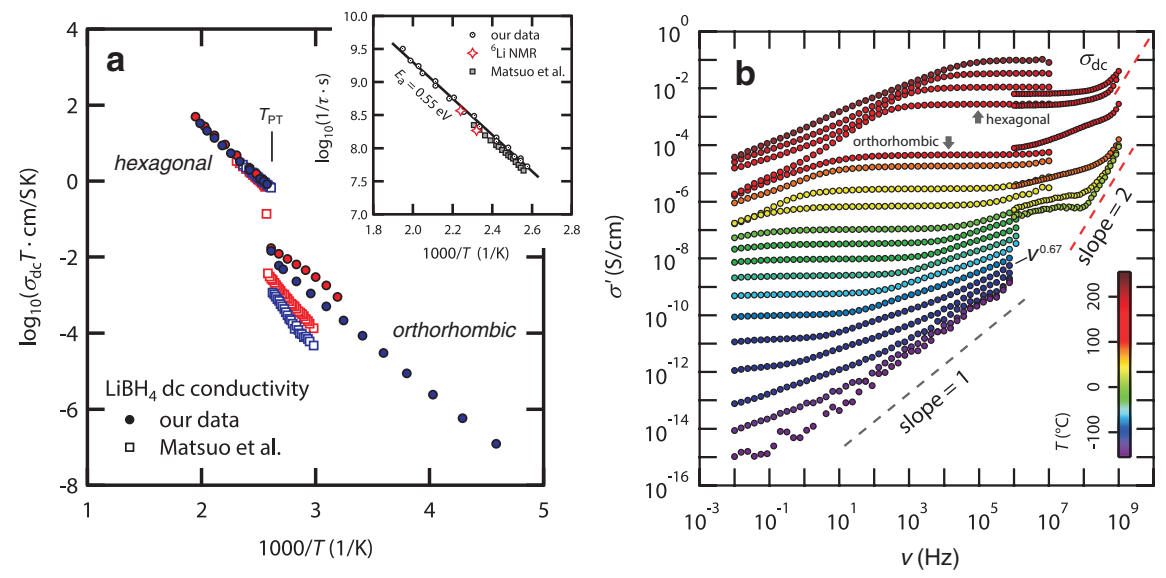

Fig. 1: (a) Temperature dependence of the ionic conductivity of $\mathrm{LiBH}_{4}$ plotted as $\sigma_{\mathrm{dc}} T \mathrm{vs} .1 / T$. The conductivity jumps up at the temperature where the phase transformation takes place. Circles: our data; squares: data from literature, see Matsuo et al. [45]; symbols in blue refer to the cooling step while symbols in red illustrate the change of $\sigma_{\mathrm{dc}} T(1 / T)$ during heating the sample. Inset: $\mathrm{Li}^{+}$jump rates either obtained from ${ }^{6} \mathrm{Li}$ SLR NMR, see Fig. 2a, or via converting $\sigma_{\mathrm{dc}}$ into $1 / \tau$ using the Nernst-Einstein and Einstein-Smoluchowski equation, see Ref. [7] for details. (b) Underlying conductivity isotherms recorded for orthorhombic and hexagonal $\mathrm{LiBH}_{4}$. From the frequency independent plateaus we read off the $\sigma_{\mathrm{dc}}$ values. The dispersive regimes can be approximated with power laws, $\sigma^{\prime} \propto v^{n}$ whose exponent $n$ varies from 1 to 2 . While $n=1$ has been frequently ascribed to strictly localized motions, $n=2$, which is observed in the $\mathrm{GHz}$ regime, might be caused by the effect of lattice vibrations on the isotherms. Considering those recorded around $273 \mathrm{~K}$ two plateau regions are visible. The one at higher frequencies might be ascribed to bulk ion dynamics while that at lower $v$ also contains the influence of grain boundaries somewhat hindering ion transport over long distances. See Ref. [28] for further discussion.

in the layered variant. The lines meet all the expectations of a system with fast diffusing spin-3/2 quadrupolar nuclei that are exposed to a mean electric field gradient. The jump in Li ion diffusivity, occurring when going from orthorhombic to hexagonal $\mathrm{LiBH}_{4}\left(P 6_{3} m c\right.$ or $P 6_{3} / m m c$, see Ref. [44]), is also seen when dc conductivity, $\sigma_{\mathrm{dc}}$, values are considered. Just above the phase transformation the ionic conductivity rapidly rises by two orders of magnitude, see Figure 1 and the conductivity isotherms in (b), $[43,45,46]$.

To decide whether Li diffusion in layer-structured, hexagonal $\mathrm{LiBH}_{4}$ is indeed of two-dimensional nature, we recorded diffusion-induced SLR NMR rates. We could gather the high-temperature flank that contains the information on the dimensionality of the diffusion process (see Table 1). Since the relaxation models useful to differentiate between 2D and 3D diffusion were developed for dipolar interactions, we focused on ${ }^{6} \mathrm{Li}$ NMR. Although the ${ }^{6} \mathrm{Li}$ nucleus is a quadrupolar one 

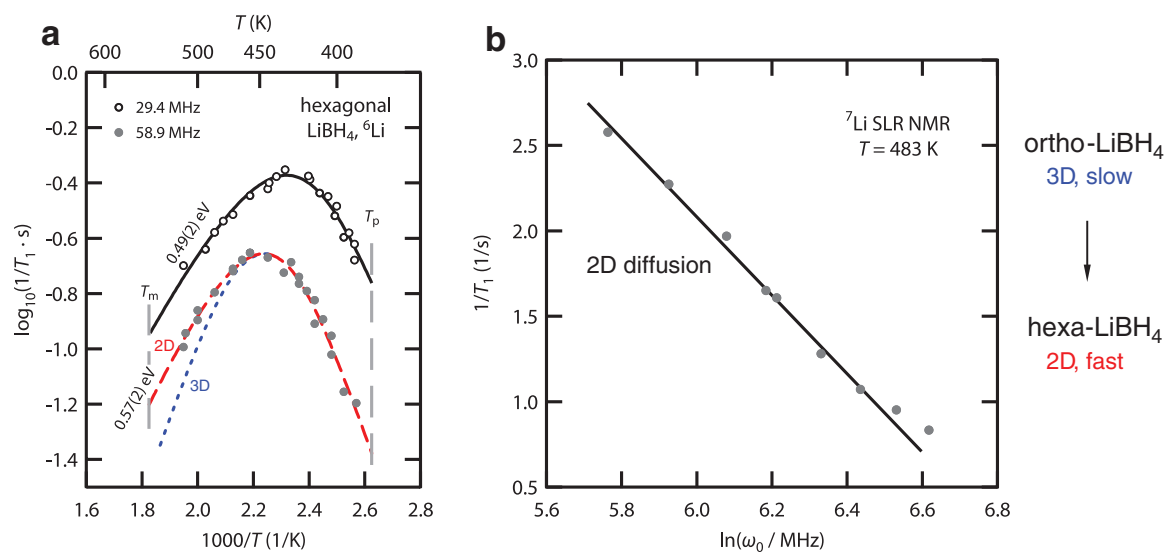

Fig. 2: (a) ${ }^{6} \mathrm{Li}$ NMR SLR rates of hexagonal $\mathrm{LiBH}_{4}$ recorded at two different Larmor frequencies ( 29.4 and $58.9 \mathrm{MHz}$ ); the rates were recorded with the saturation recovery pulse sequence. Solid lines show fits according to the model of Richards developed for 2D diffusion. Note the slight asymmetry of the rate peaks as well as the dispersive region in the high- $T$ limit. According to the relaxation model the slope of the high- $T$ flank is by a factor of 0.75 smaller than that of the low- $T$ side. (b) Frequency dependence of the rate $1 / T_{1}$ that was recorded at constant temperature $(483 \mathrm{~K})$. The line represents a linear fit showing that the prediction of Richards [16, 47] $1 / T_{1} \propto \ln \omega_{0}$ is fulfilled. cf. Ref. [12] for further information.

with a spin-quantum number of $I=1$, its quadrupole moment is much lower, by a factor of 50 , than that of ${ }^{7} \mathrm{Li}(I=3 / 2)$. Therefore, it can be regarded as a quasi spin-1/2 nucleus that is mainly subjected to dipole-dipole interactions. In Figure $2 \mathrm{a}$ the ${ }^{6} \mathrm{Li}$ NMR spin-lattice relaxation rate peaks are shown that illustrate low-dimensional Li ion diffusion in hexagonal $\mathrm{LiBH}_{4}$. As expected, the rate peaks shift toward higher $T$ the higher the Larmor frequency chosen to record the rates. Most importantly, besides an almost quadratic frequency dependence on the low- $T$ side, the rates of the high- $T$ flank, where $\omega_{0} \tau \gg 1$ holds, do not coincide as it would be expected for 3D diffusion. Instead, both rate peaks could be satisfactorily parameterized with the semi-empirical model introduced by Richards $[16,47]$. This model has been developed for 2D diffusion. It takes into account a smaller slope of the high- $T$ flank. For comparison, uncorrelated 3D motion $[5,14]$ would lead to symmetric rate peaks (see the dashed line in Figure 2a). The model of Richards predicts a logarithmic frequency dependence of the diffusion-induced SLR NMR rates in the limit $\omega_{0} \tau \gg 1$. In order to prove this prediction we recorded the rate $1 / T_{1}$ as a function of frequency at $483 \mathrm{~K}$, i.e. on the high- $T$ side of the diffusion-induced rate peaks. The result is shown in Figure $2 b$, the rate indeed follows a linear behavior if $1 / T_{1}$ is plotted vs. $\ln$ $\omega_{0}$. This behavior definitely proves the validity of the model of Richards and the $2 \mathrm{D}$ nature of $\mathrm{Li}$ ion diffusion in layer-structured $\mathrm{LiBH}_{4}$. 
Recently, the 2D diffusion process was also confirmed by theory, see, for example the ab initio molecular dynamics study performed by Aeberhard et al. [48] stating that $\mathrm{Li}$ diffusion in $\mathrm{LiBH}_{4}$ is effectively two dimensional in nature. Density functional theory calculations and quasi-elastic neutron scattering experiments of Myrdal et al. [49] also showed that the most favorable mechanism for $\mathrm{Li}^{+}$conduction takes place in the hexagonal plane; their calculations revealed that jump processes with a component in the $z$-direction have to be characterized by higher energy barriers, thus supporting the idea of a two-dimensional transport process.

As a last remark, we would like to draw the reader's attention once again to the fact that we found indications for uncorrelated Li diffusion [28]. The solid lines in Figure 2a include a quadratic frequency dependence in the low- $T$ regime. This proportionality, $1 / T_{1} \propto \omega_{0}^{-2}$, is predicted by the BPP model that is, as mentioned above, based on an exponential motional correlation function $G(t)$. The activation energy $(0.57 \mathrm{eV})$ extracted from ${ }^{6} \mathrm{Li}$ SLR NMR measurements carried out at 58.9 MHz agrees well with that obtained from conductivity measurements $(0.55 \mathrm{eV})$. Therefore, we conclude that the ions are exposed to a rather regular potential landscape with no differences between short-range and long-range ion dynamics. The corresponding conductivity isotherms of hexagonal $\mathrm{LiBH}_{4}$ do not reveal a pronounced dispersive region which would be indicative of correlated jump processes [50]. Instead the conductivity plateau directly discharges into the regime where the real part of the complex conductivity is governed by lattice vibrations, i.e. characterized by $\sigma^{\prime} \propto v^{2}$, see Figure $1 \mathrm{~b}$.

\section{$2.2 \mathrm{Li}_{x} \mathrm{NbS}_{2}$}

Layer-structured transition metal chalcogenides are ideally suited to host foreign atoms such as $\mathrm{Li}^{+}$or $\mathrm{Ag}^{+}$[25]. Self-diffusion of these guest atoms is anticipated to mainly occur within the van-der-Waals gap, i.e. between the transition metal oxide or sulfide layers. For instance, earlier NMR relaxation studies revealed 2D Li ion diffusion in the layer-structured 1T-modification of $\mathrm{Li}_{x} \mathrm{TiS}_{2}$ for $x$ considerably lower than one $[11,26]$. In $\mathrm{Li}_{0.7} \mathrm{TiS}_{2}$ a single diffusion process was probed covering a dynamic range of almost 10 orders of magnitude [26, 27]. In the $3 \mathrm{R}$ as well as the $2 \mathrm{H}$ phase of $\mathrm{Li}_{x} \mathrm{NbS}_{2}$ the Li ions have also access to intralayer diffusion; its low dimensionality could be revealed via frequency-dependent ${ }^{7} \mathrm{Li} \mathrm{NMR}$ spin-lock relaxation measurements $[29,30]$. As (first-order) quadrupole interactions in $\mathrm{Li}_{x} \mathrm{NbS}_{2}$ turned out to be relatively small, the ${ }^{7} \mathrm{Li}$ nucleus served as a suitable probe for our analysis (Figure 3).

In contrast to $\mathrm{LiBH}_{4}$ for $3 \mathrm{R}-\mathrm{Li}_{0.7} \mathrm{NbS}_{2}$ much higher temperatures $(>500 \mathrm{~K})$ are, however, needed to record the high- $T$ flank of the diffusion-induced rate peaks 
a

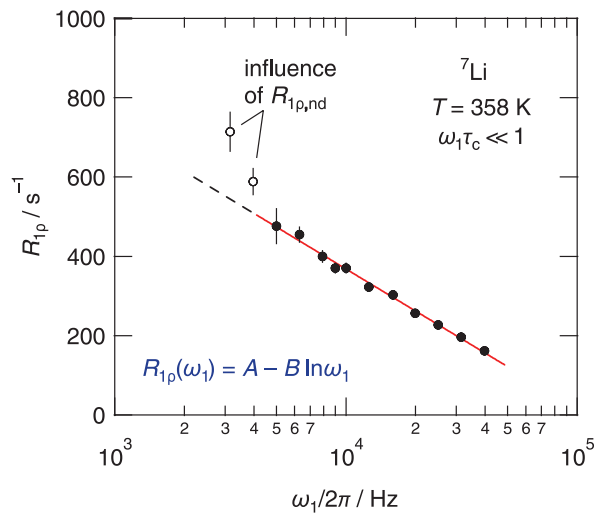

b

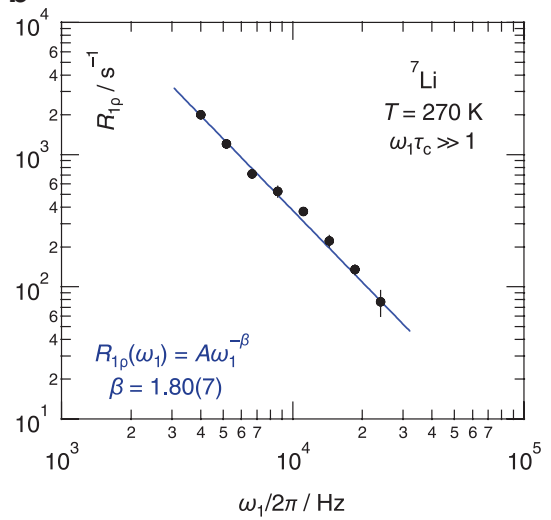

Fig. 3: Frequency dependence of the spin-lock ${ }^{7} \mathrm{Li}$ SLR NMR rate $\left(1 / T_{1 \mathrm{p}} \equiv R_{1 \mathrm{p}}\right)$ of $3 \mathrm{R}-\mathrm{Li}_{0.7} \mathrm{NbS}_{2}$; the sample was annealed at elevated $T$ to ensure a homogenous distribution of the Li ions among the vacant sites between the $\mathrm{NbS}_{2}$ sheets. (a) Diffusion-induced rates recorded at $358 \mathrm{~K}$. The rates at locking frequencies $\omega_{1} / 2 \pi$ lower than $5 \mathrm{kHz}$ are influenced by non-diffusive (nd) background effects; they were excluded from the linear fit shown. (b) The sub-quadratic frequency dependence of $R_{1 \rho}$ in the limit $\omega_{1} \tau \gg 1$. See [29] for further details.

$1 / T_{1}(1 / T)$. Therefore, we took advantage of spin-lock relaxometry that is usually carried out at locking frequencies $\omega_{1} / 2 \pi$ in the $\mathrm{kHz}$ range [29]. With $\omega_{1} / 2 \pi$ ranging from 5 to $40 \mathrm{kHz}$ the peaks $1 / T_{1 p}(1 / T)$ show up between $285 \mathrm{~K}$ and $330 \mathrm{~K}$ clearly shifting toward higher $T$ with increasing locking frequency. In Figure $3 a$ and $b$ the frequency dependence of the ${ }^{7} \mathrm{Li}$ SLR NMR rate in the limits $\omega_{1} \tau \ll 1(358 \mathrm{~K})$ and $\omega_{1} \tau \gg 1(270 \mathrm{~K})$ are shown. In the low- $T$ range we observed a slight deviation from uncorrelated motion that is expressed by the exponent $\beta$ being, for correlated motion, smaller than two. Most importantly, for $\omega_{1} \tau \ll 1$ the spin-lock SLR NMR rates linearly depend on $\ln \omega_{1}$ pointing to $2 \mathrm{D} \mathrm{Li}$ ion diffusion. As for $\mathrm{LiBH}_{4}$ the absolute change of the rates with increasing $\omega_{1}$ turned out to be measurable, but was low. This is even more pronounced for $2 \mathrm{H}_{-} \mathrm{Li}_{x} \mathrm{NbS}_{2}$ (see Figure 4). We assumed a wavelike 2D diffusion process, i.e. an oscillating trajectory which results if we consider the various sites within the van-der-Waals gap acting as transition states. Such transition states might be the tetrahedral sites connecting two octahedral voids by sharing common faces; a similar diffusion pathway was earlier found for $\operatorname{Li}_{x} \mathrm{TiS}_{2}(x \leq 0.7)$ by means of ${ }^{7} \mathrm{Li}$ stimulated echo NMR [7, 27].

For $2 \mathrm{H}-\mathrm{Li}_{x} \mathrm{NbS}_{2}$ we investigated three samples with $x=0.3,0.7$ and 1.0 [30]. ${ }^{7} \mathrm{Li}$ SLR NMR in both the laboratory and rotating frame of reference were used to quantify the effect of correlation effects on Li ion diffusion with increasing charge carrier density. If analyzing $1 / T_{1 \rho}$ with the $2 \mathrm{D}$ spectral density function 

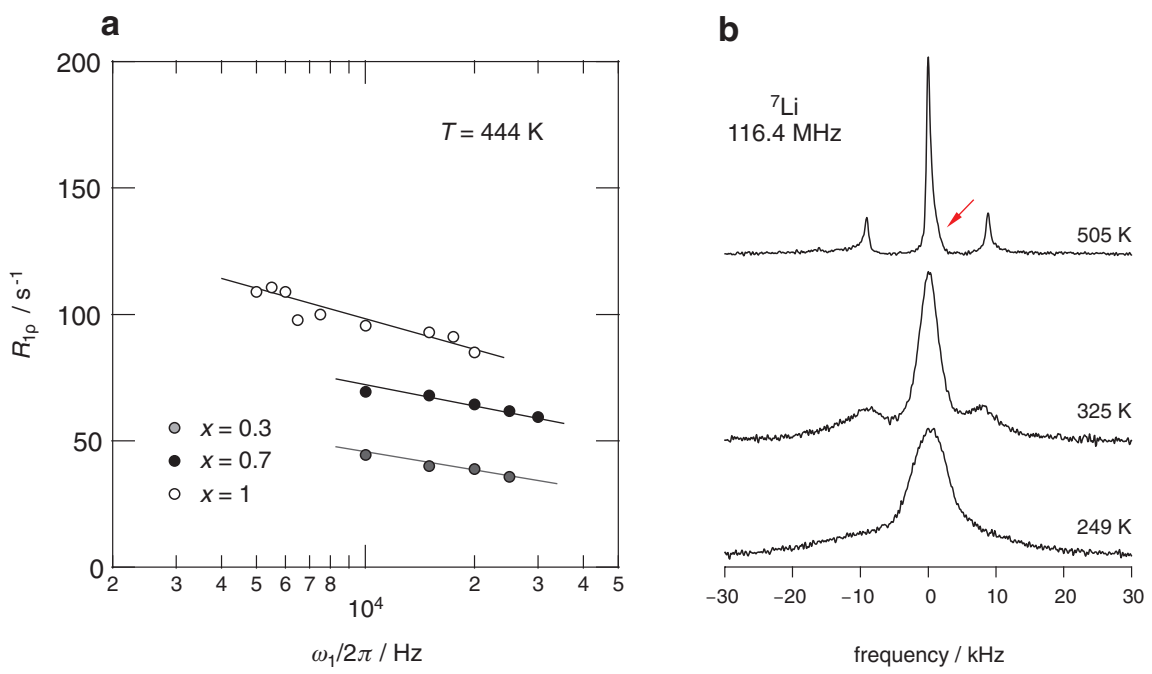

Fig. 4: (a) Frequency dependence of the ${ }^{7} \mathrm{Li} N M R$ spin-lock $S L R$ rates of $\mathrm{Li}_{0.3} \mathrm{NbS}_{2}, \mathrm{Li}_{0.7} \mathrm{NbS}_{2}$, and $\mathrm{Li}_{1.0} \mathrm{NbS}_{2} .444 \mathrm{~K}$ refers to the high- $T$ limit with $\omega_{1} \tau \ll 1$. (b) ${ }^{7} \mathrm{Li} \mathrm{NMR}$ spectra of $\mathrm{Li}_{1.0} \mathrm{NbS}_{2}$. Whereas at low $T$ the NMR line is dipolarly broadened and quadrupole interactions are seen as broad humps next to the central line, at elevated T motional averaging leads to sharp resonances. The shape of the quadrupolar powder pattern and the position of the $90^{\circ}$ singularities indicate a mean electric field gradient with axial symmetry. The quadrupole coupling constant is ca. 17 $\mathrm{kHz}$. It decreases to $11 \mathrm{kHz}$ if $x$ is decreased to $x=0.3$. The emergence of a distinct quadrupole powder pattern with fixed positions of the $90^{\circ}$ singularities on the $\mathrm{Hz}$ scale (see the spectra recorded at $325 \mathrm{~K}$ and $505 \mathrm{~K}$ ) points to an anisotropic diffusion process, most likely confined to two dimensions as suggested by $1 / T_{10}$ measurements. The small arrow highlights the (slight) asymmetry of the central line that is expected for Li ions located in the ab plane of the $\mathrm{NbS}_{2}$ structure. See Ref. [30] for further explanation.

of Richards, we were able to clearly witness that Li ion self-diffusivity is slowed down with increasing $x$ [30]. Activation energies $E_{\mathrm{a}}$ ranged from $0.44 \mathrm{eV}(x=0.3)$ and $0.48 \mathrm{eV}(x=0.7)$ to $0.64 \mathrm{eV}(x=1.0)$; accordingly, the rate peaks systematically shifted to higher $T$ the larger $x$. Considering, however, the activation energies from laboratory frame $1 / T_{1}$ in the limit $\omega_{0} \tau \gg 1$ we observed the opposite trend: At the same time, the $\beta$ parameter used to describe the asymmetry of the $1 / T_{10}$ rate peaks decreased with increasing $x[\beta=1.8,(x=0.3), \beta=1.7(x=0.7)$, and $\beta=1.4$ $(x=0.3)$ [ [30]. This observation was interpreted in terms of an increasing influence of correlation effects due to enhanced Coulomb interactions for samples with a high concentration of mobile Li ions [30]. Such effects lead to lower slopes in the low- $T$ limit than expected for ion jump diffusion. Hence, an $E_{\mathrm{a}}$ that decreases with increasing $x$ turned out to be measure for correlation effects rather than a 
parameter to describe energy barriers on the angstrom length scale. Of course, to a small extent, the widening of the van-der-Waals gap with increasing Li content might reduce the local barriers the ions have to surmount. In our case, we have, however, to take into account two facts. First, we deal with a rather regular potential landscape and second we saw that long-range ion diffusivity decreases with increasing $x$. Therefore, we could not find convincing arguments that would support the idea of enhanced local motions the higher the Li content, not even if we consider interlayer diffusion processes via defects or interstitial sites.

\section{$2.3 \mathrm{Li}_{0.17} \mathrm{SnS}_{2}$}

Apart from $\mathrm{LiBH}_{4}, \mathrm{Li}_{x} \mathrm{TiS}_{2}$ and $\mathrm{Li}_{x} \mathrm{NbS}_{2}$ the Sn-analogue $\mathrm{Li}_{0.17} \mathrm{SnS}_{2}$ was used as another model substance to probe low-dimensional diffusion [31]. In contrast to $\mathrm{Li}_{x} \mathrm{TiS}_{2}$ in $\mathrm{SnS}_{2}$ the intra- and interlayer octahedral and tetrahedral sites may act as hosts for Li ions, see Figure 5a. According to Pietrass et al. [51] the amount of Li ions inserted needs to be relatively low to avoid the occupancy of voids in the $\mathrm{SnS}_{2}$ layers. Thus, we focused on a sample with $x=0.17$; at this low Li content also

a

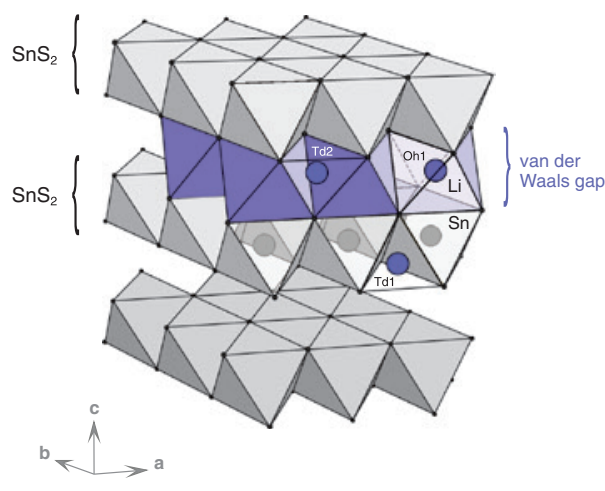

b

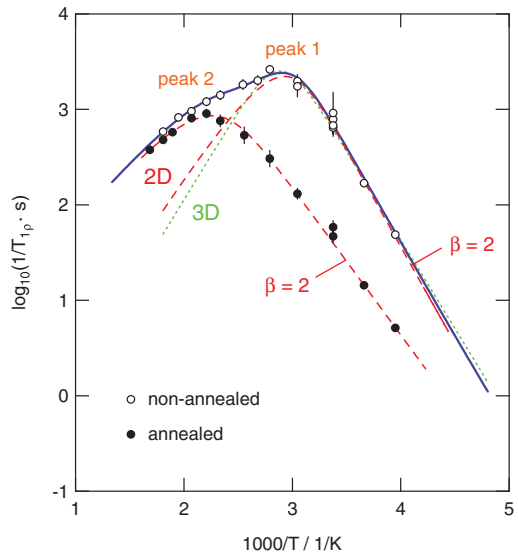

Fig. 5: (a) Crystal structure of $\mathrm{Li}_{x} \mathrm{SnS}_{2}$ with the tetrahedral sites highlighted that, besides the octahedral ones, are also able to host Li ions. (b) ${ }^{7} \mathrm{Li}$ spin-lock SLR NMR rates of $\mathrm{Li}_{0.17} \mathrm{SnS}_{2}$ before and after annealing the powder sample. While peak 2 does not change much in both amplitude and position, peak 1 is significantly reduced in amplitude. Presumably, peak 1 represents clustered Li ions near the surface regions; such clusters may form during the lithiation process, that is, the treatment of $\mathrm{SnS}_{2}$ with $n$-BuLi dissolved in hexane. See text and Ref. [31] for further explanation. 
the influence of staging effects can be ruled out [52]. As opposed to $\mathrm{Li}_{x} \mathrm{NbS}_{2}$ and $\mathrm{Li}_{x} \mathrm{TiS}_{2}$ for a non-annealed sample we observed two overlapping relaxation rate peaks $1 / T_{1 \rho}$ whose amplitudes change upon annealing the sample (see Figure $5 \mathrm{~b}$ ). We assume that peak 1 represents those ions that form clusters near the surfaceinfluenced regions of the $\mathrm{SnS}_{2}$ crystallites. In such regions the ions may have access to short-ranged diffusion pathways. Upon annealing the ions became more uniformly distributed among the available octahedral sites between the $\mathrm{SnS}_{2}$ sheets. As a consequence, peak 1 drastically loses in intensity. The final NMR rates can either be parameterized with two rate peaks or, roughly speaking, with only one rate peak that agrees with 2D diffusion (see below). Due to the complex ion dynamics observed in $\mathrm{Li}_{0.17} \mathrm{SnS}_{2}$ and the heat sensitivity of the samples we passed further frequency-dependent NMR measurements up. The 2D nature of $\mathrm{Li}$ ion diffusion in $\mathrm{Li}_{0.17} \mathrm{SnS}_{2}$, however, indirectly showed up when analyzing the NMR response before annealing.

Regarding the situation before heat treatment, peak 2 was approximated with the expression of Richards for 2D diffusion [16, 47]. For peak 2 we used either a BPP-type (3D) or 2D ansatz [31]. The overall fit is shown in Figure 5b as a thick solid line. The underlying two fits of peaks 1 and 2 are given by dashed lines. If peak 2 is approximated with the spectral density for $2 \mathrm{D}$ diffusion it automatically reflects the NMR response that is obtained after the annealing step. Note that in Figure 5b the data points for the annealed sample are shown for comparison only.

\section{$2.4 \mathrm{LiC}_{6}$}

Graphite is one of the best known 2D energy materials; it represents an excellent anode material in $\mathrm{Li}$ ion batteries. Li ions can be reversibly inserted and deinserted without any severe structural change of the host. The stage-1 compound $\mathrm{LiC}_{6}$ is a gold-colored substance. It consists of commensurate layers of carbon and lithium; Li resides in the central site below and above each third hexagon formed by $\mathrm{C}$ atoms (see Figure 6). The stacking sequence is $A \alpha A \alpha$ with a periodicity of $3.71 \AA$; $A$ denotes the graphite basal plane and $\alpha$ represents the intercalate layer. The ions have access to several hopping processes that have been the subject of some calculations on ion dynamics in graphite intercalation compounds. The host structure allows for in-plane jump diffusion, see Figure 6. In earlier studies it has been argued that diffusion perpendicular to the $c$-axis cannot be ruled out completely [53]. Vacant C sites will certainly promote interlayer Li diffusion.

Although ion dynamics in this mixed-conducting compound has been investigated in the past via quasi-elastic neutron scattering (QENS) and NMR [54-56], for example we re-investigated ion self-diffusion by means of ${ }^{7} \mathrm{Li}$ spin-spin and 


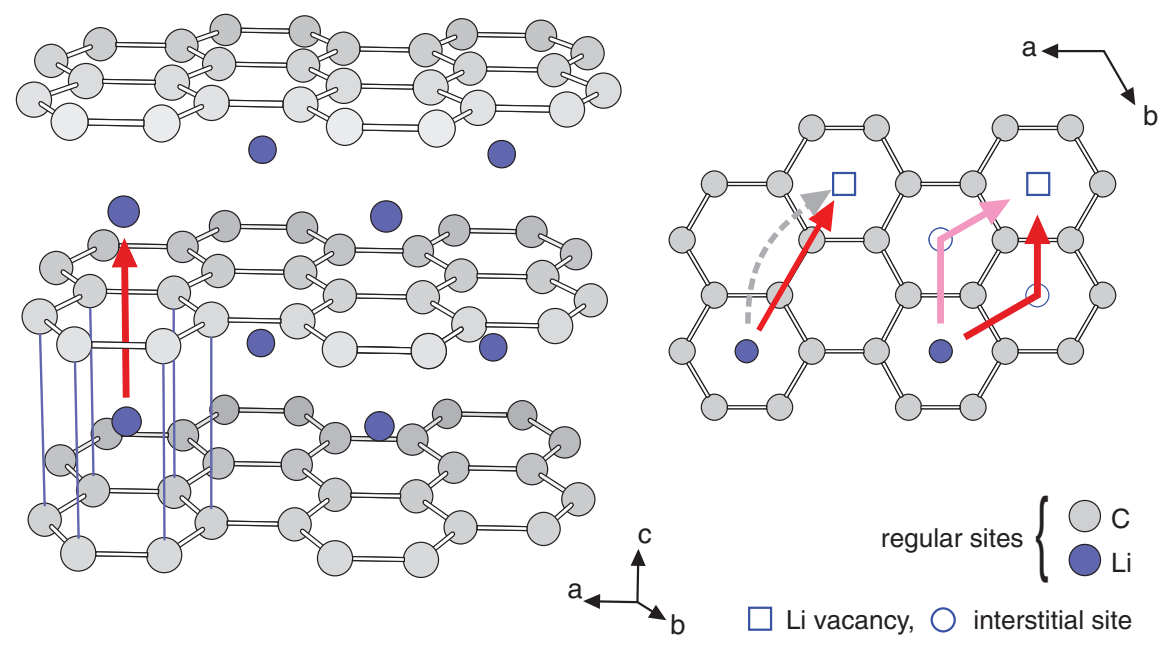

Fig. 6: Layered crystal structure of the graphite intercalation compound $\mathrm{LiC}_{6}$. Blue spheres denote $\mathrm{Li}$ ions residing between the $\mathrm{C}$ layers. The arrows represent the various diffusion pathways involving Li vacancies and interstitial sites. Diffusion perpendicular to the $c$-axis is most likely possible via point defects in the carbon lattice, see Ref. [32].

spin-lock NMR relaxometry [32]. Whereas the laboratory ${ }^{7} \mathrm{Li}$ SLR NMR rates were strongly affected by the $\mathrm{Li}^{+}$-electron interactions in $\mathrm{LiC}_{6}$ (see Figure $7 \mathrm{a}$ ), the rates in the rotating frame of reference as well as the corresponding spin-spin relaxation rates were useful to extract diffusion parameters.

The rates $1 / T_{1 p}$ pass through a diffusion-induced rate peak which is located at $314 \mathrm{~K}$. It can be best represented with a Lorentzian-shaped BPP-type spectral density function. The asymmetry parameter turned out to be ca. 1.7. For $E_{\mathrm{a}}$ we obtained $0.57 \mathrm{eV}$; the activation energy of the low- $T$ side is only $0.42 \mathrm{eV}$ indicating correlated motion [32]. This value is comparable with that from ${ }^{7} \mathrm{Li}$ NMR spin-spin relaxation $(0.53 \mathrm{eV})$ and agrees well with the result from Figure $7 \mathrm{~b}$. In Figure $7 \mathrm{~b}$ Li jump rates from the various NMR methods, including previous results from ${ }^{8} \mathrm{Li} \beta$-NMR on oriented $\mathrm{LiC}_{6}$ crystals [54, 55], and QENS [56] are shown in a joint Arrhenius plot. The Arrhenius line drawn is characterized by $0.55 \mathrm{eV}$; this activation energy agrees well with results from theory for both the in-plane vacancy and interstitial Li migration mechanism [57, 58]. Residence times in the order of $1 \mu \mathrm{s}$ are obtained at ca. $350 \mathrm{~K}$. At temperatures below the melting point of water we have to deal with residence times in the order of $10^{4} \mathrm{~s}^{-1}$. Such information has enormous practical importance. At low $T$ the slow kinetics and the low $\mathrm{Li}^{+}$diffusion coefficients in graphite battery anodes promote Li plating rather than $\mathrm{Li}$ insertion. The deposition of metallic Li on the graphite surface is highly problematic in terms of safety and life cycle of these storage systems. ${ }^{7} \mathrm{Li}-\mathrm{NMR}$ and 
a

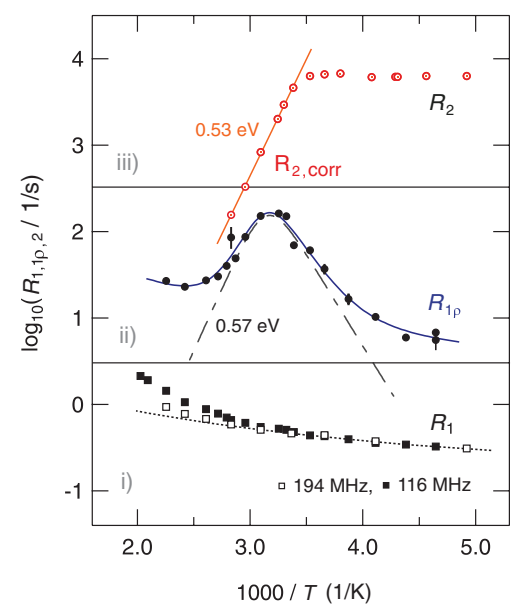

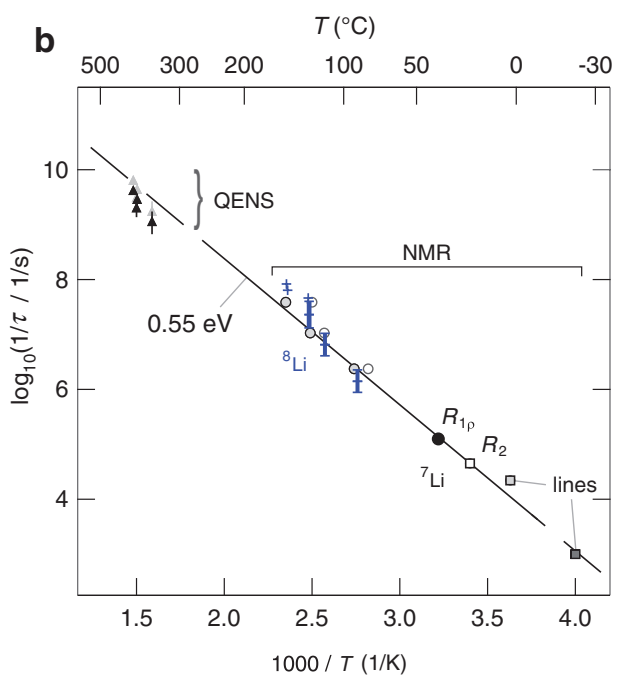

Fig. 7: (a) Temperature dependence of the various types of ${ }^{7} \mathrm{Li}$ NMR relaxation rates of $\mathrm{LiC}_{6}$ : (i) SLR in the laboratory frame recorded at two different resonance frequencies. (ii) $1 / T_{1 p}$ relaxation rates recorded at a locking frequency of $20 \mathrm{kHz}$. (iii) $1 / T_{2}$ spin-spin relaxation rates showing Arrhenius-type behavior at temperatures higher than $285 \mathrm{~K}$. (b) Li jump rates deduced from ${ }^{7} \mathrm{Li}$ NMR [32], ${ }^{8}$ Li $\beta$-NMR [54] and QENS [56]. The straight line represents an Arrhenius fit yielding $0.55 \mathrm{eV}$. At ambient the jump rate is in the order of $10^{5} \mathrm{~s}^{-1}$. See Ref. [32] for further details.

impedance studies dealing with practical aspects of graphite anode materials are, e.g. presented in Refs. [59, 60].

\section{Li compounds proffering one-dimensional diffusion}

\section{$3.1 \mathrm{Li}_{12} \mathrm{Si}_{7}$}

In polycrystalline $\mathrm{Li}_{12} \mathrm{Si}_{\vec{p}}$, being one of the Li-rich Zintl phases in the binary Li-Si system, strong evidence for one-dimensional Li motion has been reported [10, 38-40]. This $1 \mathrm{D} \mathrm{Li}^{+}$motion can be linked to channel-like columns formed by the stacked planar silicon rings which guide the Li ions in the close proximity of the channels. The Li ions sandwiched between the rings are much less mobile as has been verified by ${ }^{6} \mathrm{Li} 2 \mathrm{D}$ exchange NMR [39]. These ions can be identified by their characteristic isotropic chemical shift of $-17.2 \mathrm{ppm}$ in ${ }^{6} \mathrm{Li}$ magic angle 

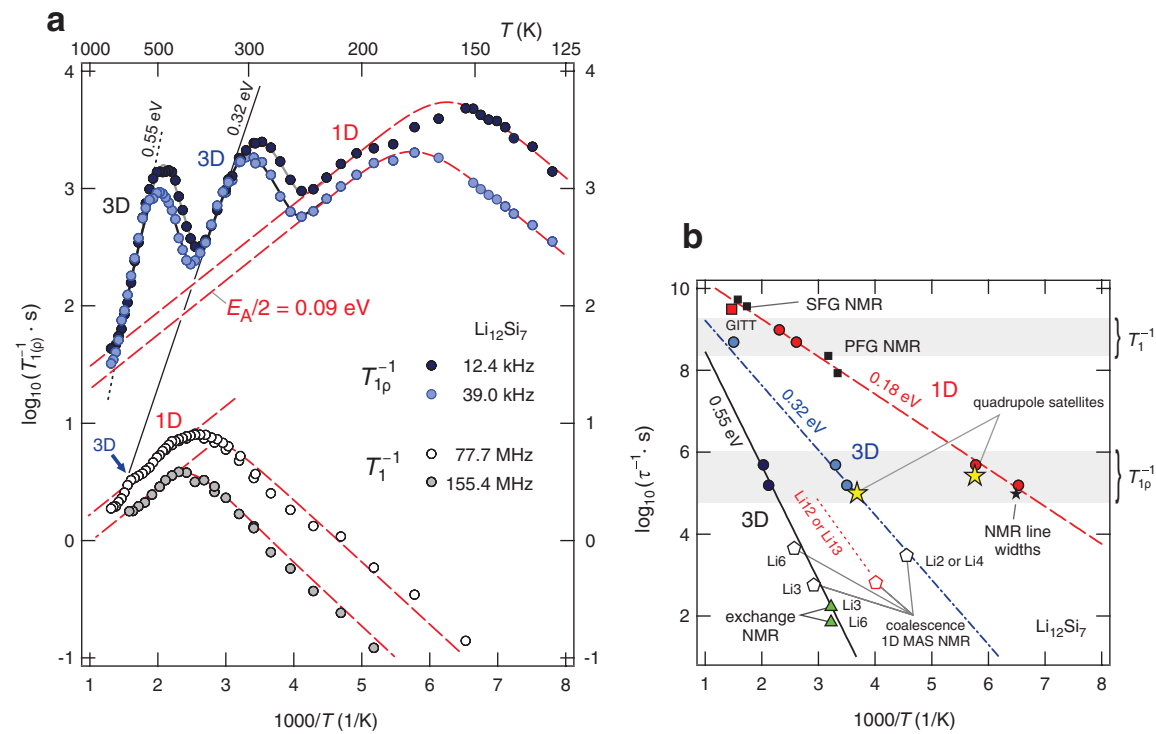

Fig. 8: (a) Temperature dependence of the diffusion-induced ${ }^{7} L i$ NMR SLR rates in the laboratory frame $\left(1 / T_{1}\right)$ and rotating frame $\left(1 / T_{10}\right)$ of polycrystalline $\mathrm{Li}_{12} \mathrm{Si}_{7}$ at two frequencies each. (b) Arrhenius plot of the Li jump rates collected from NMR relaxometry, field-gradient (FG) NMR, and other NMR methods including high-resolution techniques as well as from galvanostatic intermittent titration technique (GITT). See Refs. [39, 40] for further details.

spinning (MAS) NMR. The, at first glance, unexpected shift to a higher magnetic field has been explained by the strong shielding effects caused by an aromatic ring current of the $\mathrm{Si}_{5}$ rings [38].

In Figure 8a, the diffusion-induced ${ }^{7} \mathrm{Li}$ NMR SLR rates $1 / T_{1}$ and $1 / T_{1 p}$ are plotted versus the inverse temperature for different Larmor frequencies $\omega_{0}$ and spin-lock angular frequencies $\omega_{1}$, respectively. The spin-lock SLR rates clearly reveal at least three maxima, one being already visible at very low temperatures. In line with relaxation models for 1D diffusion (cf. Table 1) the activation energy of the high$T$ flank is given by $E_{\mathrm{A}} / 2=0.09 \mathrm{eV}$, that is, half the value of the activation energy obtained in the regime $\omega_{0} \tau \gg 1$. Together with the frequency dependence observed this asymmetry represents a strong indication of $1 \mathrm{D}$ diffusion. This quasi $1 \mathrm{D} \mathrm{Li}$ motion is also seen in our relaxometry measurements performed in the laboratory frame of reference, see Figure 8a; the corresponding diffusion-induced ${ }^{7} \mathrm{Li}$ SLR NMR rate peaks also reveal a dispersive behavior in the limit $\omega_{0} \tau \ll 1$. Taking $1 / T_{1}\left(\omega_{0}\right)$ and $1 / T_{1 p}\left(\omega_{1}\right)$ together, the data fulfill the square root frequency dependence (cf. Table 1) over three orders of magnitude [40]. An analogous result for 2D Li diffusion was early found in the case of the layered model system $\operatorname{Li}_{x} \mathrm{TiS}_{2}[5,11]$. 
The other spin-lock SLR rate peaks showing up at higher $T$ have been assigned to two different 3D Li diffusion processes being consecutively activated with increasing temperature. As is diagnostic for 3D diffusion they are symmetric in shape and show no frequency dependence on the respective high- $T$ flanks. The corresponding activation energies turned out to be $0.32 \mathrm{eV}$ and $0.55 \mathrm{eV}$.

In Figure $8 \mathrm{~b}$ the jump rates deduced from the various NMR rate peak maxima are compared with results from other NMR investigations; these techniques include the analysis of NMR MAS spectra, NMR exchange spectroscopy, the analysis of ${ }^{7} \mathrm{Li}$ NMR quadrupolar satellites and ${ }^{7} \mathrm{Li}$ NMR line widths. Moreover, Li diffusion coefficients from static (SFG) and pulsed field-gradient (PFG) NMR as well as electrochemical investigations (galvanostatic intermittent titration technique, GITT) are shown. The diffusion coefficients measured by ${ }^{7}$ Li SFG NMR and PFG NMR were converted to jump rates using the EinsteinSmoluchowski equation. FG NMR shows that the 1D Li motion observed with ${ }^{7} \mathrm{Li}$ NMR relaxometry is a fast, long-range diffusion process. In a recent study [39] it has been shown that in $\mathrm{Li}_{12} \mathrm{Si}_{7} 9$ of $13 \mathrm{Li}$ positions contribute to this jump process. The other two diffusion processes, which are 3D in nature, correspond to Li ions being stronger bound to the Si lattice and having medium and low diffusivity, respectively [38]. Our experimental findings were recently corroborated in a dedicated atomistic study using density functional theory by Shi et al., see Ref. [61].

\section{$3.2 \mathrm{Li}_{0.7} \mathrm{Nb}_{3} \mathrm{~S}_{4}$}

In contrast to $\mathrm{Li}_{12} \mathrm{Si}_{7}$ with its multiple Li sites, in $\mathrm{Li}_{x} \mathrm{Nb}_{3} \mathrm{~S}_{4}$ [62] the guest ions solely reside in the channels formed by the Nb-S units. Li motion is supposed to take place mainly within these channels. For isostructural $\mathrm{Li}_{0.7} \mathrm{Nb}_{3} \mathrm{Se}_{4}$, Kriger et al. [63] found indications for inter-channel and intra-channel $\mathrm{Li}$ hopping. We employed ${ }^{7} \mathrm{Li}$ NMR SLR to study the effects of dimensionality on Li motion in $\mathrm{Li}_{0.7} \mathrm{Nb}_{3} \mathrm{~S}_{4}$.

In Figure 9a, the ${ }^{7} \mathrm{Li}$ NMR SLR $1 / T_{1}$ and spin-lock SLR rates $1 / T_{1 \rho}$, which were recorded at the frequencies indicated, are plotted versus the inverse temperature. For the laboratory-frame SLR rates $1 / T_{1}$, no maximum could be detected; with the setup used only the low-temperature flank was reached. To obtain the diffusioninduced SLR rates, the total SLR rates were corrected for the non-diffusive background rates whose temperature dependence was approximated by a power law $T^{-\alpha}$ with $\alpha \approx 2$. The slope of the low- $T$ flank of the purely diffusion-induced SLR rate yielded an activation energy of $E_{\mathrm{A}}=0.25(2) \mathrm{eV}$, which we attribute to shortrange Li-ion movements. Most likely, the slight change in slope at $440 \mathrm{~K}$ indicates a change in diffusion mechanism. 
a

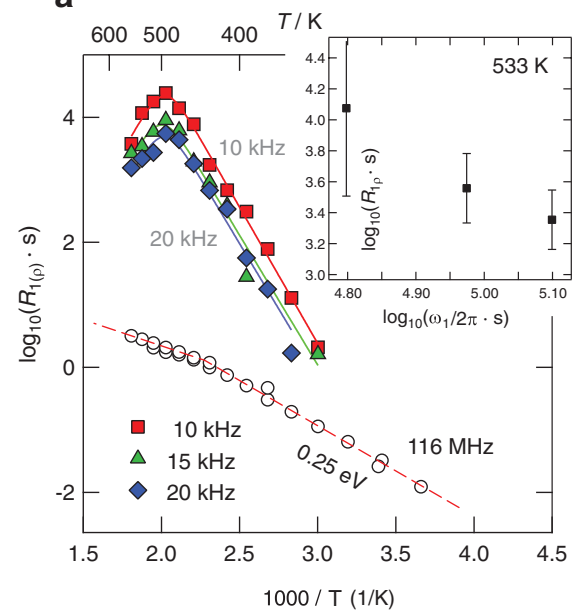

b

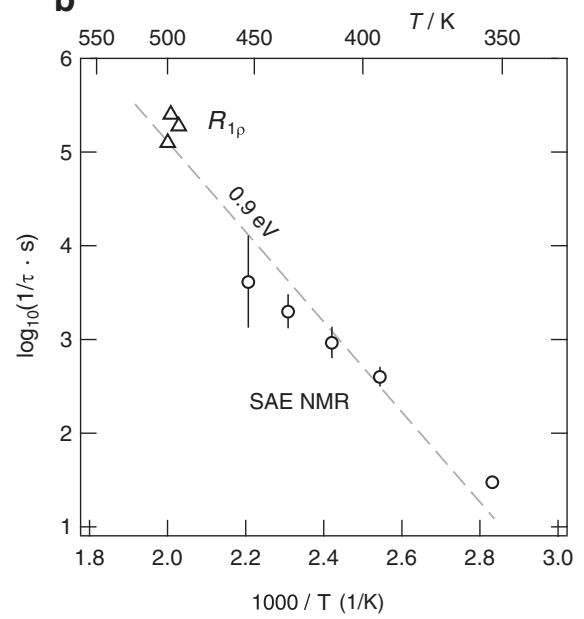

Fig. 9: (a) Diffusion-induced ${ }^{7} \mathrm{Li}$ NMR SLR $1 / T_{1}$ and spin-lock SLR rates $1 / T_{1 \rho}$ of $\mathrm{Li}_{0.7} \mathrm{Nb}_{3} \mathrm{~S}_{4}$; the $\mathrm{SLR}$ rates were recorded at $116 \mathrm{MHz}$ and at spin-lock frequencies $\omega_{1} / 2 \pi$ of 10,15 , and $20 \mathrm{kHz}$ in the temperature range of $170-550 \mathrm{~K}$. Continuous lines correspond to the BPP model to obtain the exact position of the peak maximum and additionally the activation energy of $0.65(6) \mathrm{eV}$. The low-temperature flank of the SLR rates $1 / T_{1}$ shows Arrhenius behavior and the fit gave an activation energy of $0.25(2) \mathrm{eV}$. Inset: Double-logarithmic plot of the spin-lock ${ }^{7}$ Li NMR rates $1 / T_{1 p}$ versus frequency $\omega_{1} / 2 \pi$ for the case $\omega_{1} \tau \ll 1$ at $533 \mathrm{~K}$. A frequency dependence was observed on the high-temperature flank, which indicates low-dimensional Li motion. (b) Li jump rates of $\mathrm{Li}_{0.7} \mathrm{Nb}_{3} \mathrm{~S}_{4}$ obtained from the ${ }^{7} \mathrm{Li}$ NMR $1 / T_{1 \rho}$ maxima and from ${ }^{7} \mathrm{Li}$ NMR SAE measurements. The dashed line corresponds to an activation energy of $0.9 \mathrm{eV}$ and a pre-factor of $1 \times 10^{14} \mathrm{~s}^{-1}$.

Information on slower $\mathrm{Li}$ diffusion processes in $\mathrm{Li}_{0.7} \mathrm{Nb}_{3} \mathrm{Se}_{4}$ were obtained by spin-lock ${ }^{7} \mathrm{Li}$ SLR NMR relaxometry for frequencies of $10 \mathrm{kHz}, 15 \mathrm{kHz}$, and $20 \mathrm{kHz}$, respectively. The corresponding rates were also corrected by the power law for any non-diffusive background effects; $\alpha$ ranged from 1 to 2.5. For all three frequencies, relaxation rate maxima were observed. The continuous lines show fits with a BPP-type relaxation model used to calculate the position of the peaks and to estimate the activation energy in the regime $\omega_{1} \tau \gg 1$. Here, the fits yielded $E_{\mathrm{A}}=0.65(6) \mathrm{eV}$; such a relatively large activation energy might indeed be attributed to channel-like Li diffusion. Note that activation energies deduced in the limit $\omega_{0} \tau \gg 1$ can be influenced by correlation effects reducing the slope of the rate peak in this limit.

The view of slow spatially restricted Li diffusion is supported by the doublelogarithmic plot of the diffusion-induced spin-lock NMR SLR rate vs. the locking frequency $(533 \mathrm{~K})$. The frequency dependence observed is not in contradiction to a slow 1D low-diffusion process in $\mathrm{Li}_{0.7} \mathrm{Nb}_{3} \mathrm{~S}_{4}$ [see inset of Figure 9a]. 
Li jump rates were also determined by ${ }^{7} \mathrm{Li}$ NMR spin-alignment echo (SAE) measurements; the corresponding decay rates are shown together with the results from spin-locking ${ }^{7} \mathrm{Li}$ NMR in Figure 9b. If taken together an activation energy of ca. $0.9 \mathrm{eV}$ is obtained clearly pointing to sluggish $\mathrm{Li}$ ion transport. The pre-factor of the underlying Arrhenius law is in the order of $10^{14} \mathrm{~s}^{-1}$. This value is in the order of phonon frequencies and, thus, comparable to those of other Li ion conductors such as $\mathrm{Li}_{3} \mathrm{~N}, \mathrm{Li}_{4} \mathrm{Ti}_{5} \mathrm{O}_{12}, \mathrm{Li}_{7} \mathrm{BiO}_{6}$, or $h-\mathrm{Li}_{0.7} \mathrm{TiS}_{2}[27,64-68]$.

\subsection{Mixed-alkali $\mathrm{Li}_{x} \mathrm{Na}_{2-x} \mathrm{Ti}_{6} \mathrm{O}_{13}$}

Another potential candidate to observe one-dimensional $\mathrm{Li}$ diffusion is $\mathrm{Li}_{2} \mathrm{Ti}_{6} \mathrm{O}_{13}$. In contrast to $\mathrm{Li}_{0.7} \mathrm{Nb}_{3} \mathrm{~S}_{4}, \mathrm{Li}_{2} \mathrm{Ti}_{6} \mathrm{O}_{13}$ does not act as a classical intercalation material. $\mathrm{Li}$ insertion occurs through an ion exchange reaction using the parent compound $\mathrm{Na}_{2} \mathrm{Ti}_{6} \mathrm{O}_{13}$ [69]. The Li ion occupies the same sites as $\mathrm{Na}$ and the two crystallographic sites available are identical. ${ }^{23} \mathrm{Na}$ and ${ }^{6} \mathrm{Li}$ as well as ${ }^{7} \mathrm{Li} \mathrm{NMR}$ spectra show only a single line. The end members $\mathrm{Na}_{2} \mathrm{Ti}_{6} \mathrm{O}_{13}$ and $\mathrm{Li}_{2} \mathrm{Ti}_{6} \mathrm{O}_{13}$ differ in thermal stability. Whereas $\mathrm{Na}_{2} \mathrm{Ti}_{6} \mathrm{O}_{13}$ is stable at least up to $1273 \mathrm{~K}$ [33], $\mathrm{Li}_{2} \mathrm{Ti}_{6} \mathrm{O}_{13}$ is only stable below $873 \mathrm{~K}[70]$.

The ${ }^{23} \mathrm{Na}$ and ${ }^{7} \mathrm{Li}$ NMR SLR rates measured are shown in Figure 10 covering a temperature range from 295 to $870 \mathrm{~K}$. ${ }^{23} \mathrm{Na}$ NMR SLR rates were recorded under MAS conditions using a spinning rate of $3 \mathrm{kHz}$. Any influence of spinning on the SLR rates was excluded; in general, such influence has been found to be low up to $5 \mathrm{kHz}$ spinning frequency for ${ }^{1} \mathrm{H}$ NMR SLR [72]. The ${ }^{23} \mathrm{Na}$ NMR SLR rates were corrected for the non-diffusive background effects using a power law $T^{\alpha}$, as already discussed above. The exponent $\alpha=1.1(2)$ indicates an interaction of ${ }^{23} \mathrm{Na}$ with paramagnetic centers or conduction band electrons. Most likely, a change in the relaxation mechanism shows up at higher temperatures, which led to a further increase of the NMR SLR rates. In addition to the interactions with paramagnetic centers and conduction band electrons being dominant at low temperatures, it might be possible that spin-phonon coupling through quadrupolar interaction induces a further contribution to non-diffusive background relaxation at the highest temperatures [73].

After taking into account any background effects, diffusion-induced ${ }^{23} \mathrm{Na}$ NMR SLR rates were obtained. Due to the larger quadrupolar moment of ${ }^{23} \mathrm{Na}$ compared to ${ }^{7} \mathrm{Li}$, SLR is mainly caused by quadrupolar interactions of the ${ }^{23} \mathrm{Na}$ nuclei with electric field gradients at their sites. From the ${ }^{23} \mathrm{Na}$ SLR rate peak the Na jump rate in $\mathrm{Na}_{2} \mathrm{Ti}_{6} \mathrm{O}_{13}$ was calculated to be $\tau^{-1} \approx 1 \times 10^{9} \mathrm{~s}^{-1}$ at $692 \mathrm{~K}$. The activation energy was estimated to be ca. $0.82 \mathrm{eV}$.

${ }^{7} \mathrm{Li}$ NMR SLR rates were analogously corrected for the non-diffusive background. The power law exponent turned out to be $\alpha=4$.2, i.e. much higher than 

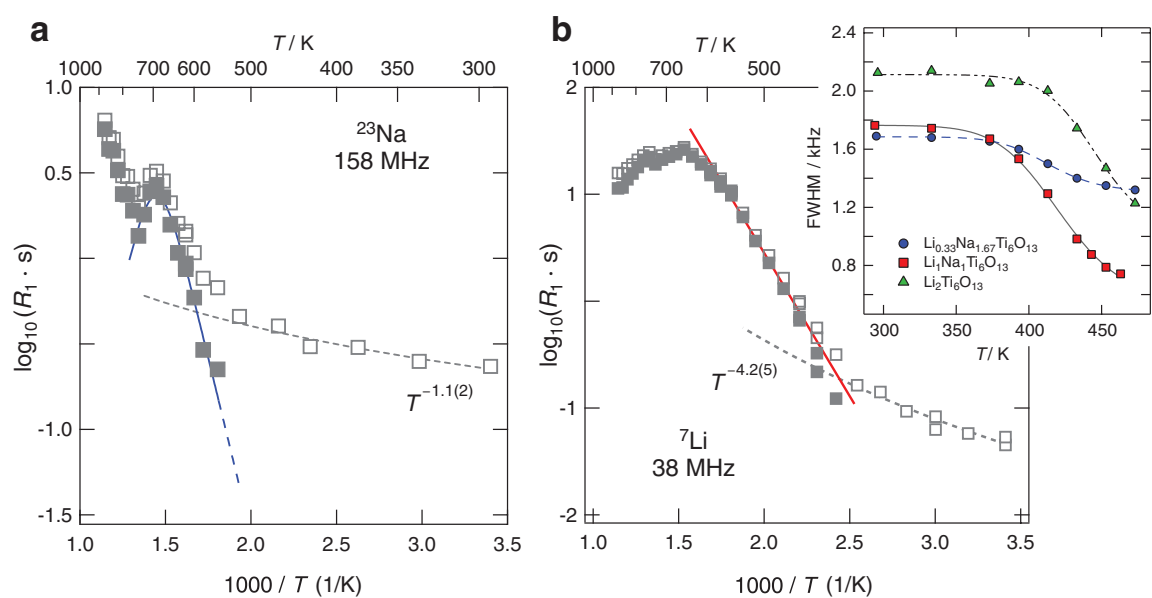

Fig. 10: (a) ${ }^{23} \mathrm{Na}$ NMR SLR rates of $\mathrm{Na}_{2} \mathrm{Ti}_{6} \mathrm{O}_{13}$ recorded at a Larmor frequency of $158 \mathrm{MHz}$ using the saturation recovery pulse sequence. A commercial high-temperature probe (Bruker BioSpin $\mathrm{GmbH}$, LASMAS) was used. The sample was prepared in a $7 \mathrm{~mm}$ rotor and heated by a IR laser system. The sample was rotated with a rate of $3 \mathrm{kHz}$. The temperature-dependent SLR rates exhibited a non-diffusive contribution at low temperatures (open symbols). The diffusion-induced SLR rates are shown with filled symbols. The solid line uses a BPP-fit to guide the eye. (b) ${ }^{7} \mathrm{Li}$ NMR SLR rates of $\mathrm{Li}_{2} \mathrm{Ti}_{6} \mathrm{O}_{13}$ recorded at a Larmor frequency of $\omega_{0} / 2 \pi=38 \mathrm{MHz}$. The $\mathrm{Li}_{2} \mathrm{Ti}_{6} \mathrm{O}_{13}$ sample was fire-sealed in an evacuated quartz glass tube and the static SLR measurements were performed in a home-built high-temperature probe. Again, a non-diffusive contribution to the SLR rates shows up at low $T$ (open symbols) and was corrected for by fitting a power-law function and subtraction (filled symbols). Inset: ${ }^{7} \mathrm{Li}$ NMR motional narrowing for $\mathrm{Li}_{x} \mathrm{Na}_{2-x} \mathrm{Ti}_{6} \mathrm{O}_{13}$. The line width or full width at half-maximum (FWHM) was read off at the ${ }^{7}$ Li NMR central transition for each temperature. The data was fitted by the empirical formula by Hendrickson and Bray [71] to estimate activation energies for each composition. For $\mathrm{Li}_{0.33} \mathrm{Na}_{1.67} \mathrm{Ti}_{6} \mathrm{O}_{13}$ the activation energy was calculated to be $0.8 \mathrm{eV}$, values of $0.7 \mathrm{eV}$ and $0.9 \mathrm{eV}$ were obtained for $\mathrm{LiNaTi}_{6} \mathrm{O}_{13}$ and $\mathrm{Li}_{2} \mathrm{Ti}_{6} \mathrm{O}_{13}$, respectively.

in case of the ${ }^{23} \mathrm{Na}$ NMR SLR rates. Presumably, spin-phonon couplings govern non-diffusive background SLR in $\mathrm{Li}_{2} \mathrm{Ti}_{6} \mathrm{O}_{13}$ already at low temperatures. Considering the SLR rate peak we end up with $\tau^{-1} \approx 2.4 \times 10^{8} \mathrm{~s}^{-1}$ at $674 \mathrm{~K}$. For the activation energy a value of $0.65 \mathrm{eV}$ could be estimated from the low- $T$ flank. Future studies should include frequency-dependent measurements to collect information on the dimensionality of the slow diffusion process seen by both ${ }^{23} \mathrm{Na}$ and ${ }^{7} \mathrm{Li} \mathrm{NMR}$.

Nevertheless, first hints to low-dimensional ion motion might already be obtained from the local ionic movement in mixed $\mathrm{Li} / \mathrm{Na}$ ion conductors $\mathrm{Li}_{x} \mathrm{Na}_{2-x} \mathrm{Ti}_{6} \mathrm{O}_{13}$. Static ${ }^{7} \mathrm{Li}$ NMR spectra were recorded at different temperatures for $\mathrm{Li}_{0.33} \mathrm{Na}_{1.67} \mathrm{Ti}_{6} \mathrm{O}_{13}$, LiNaTi $\mathrm{O}_{13}$, and $\mathrm{Li}_{2} \mathrm{Ti}_{6} \mathrm{O}_{13}$. These spectra showed only the central transition and with increasing temperature narrowing of the line width sets in [see inset of Figure 10b]. Line narrowing is caused by the averaging of homonuclear 
and heteronuclear dipole-dipole interaction. Motional narrowing slightly depends on $x$. With increasing Li content, particularly if we consider the samples LiNaTi ${ }_{6} \mathrm{O}_{13}$ and $\mathrm{Li}_{2} \mathrm{Ti}_{6} \mathrm{O}_{13}$, a slight increase in dipolar coupling is observed which can be explained by weaker homonuclear couplings, see van Vleck's formula [74] which was introduced to relate the spin-spin distances with the second moment of the NMR line. For $\mathrm{LiNaTi}_{6} \mathrm{O}_{13}$ almost the complete motional narrowing curve is seen, the residual line width in the range of extreme narrowing amounts to ca. $700 \mathrm{~Hz}$. The same value, although the curve is only partly detectable with our setup, is assumed for the Li-rich compound $\mathrm{Li}_{2} \mathrm{Ti}_{6} \mathrm{O}_{13}$. For $\mathrm{Li}_{2} \mathrm{Ti}_{6} \mathrm{O}_{13}$ the onset of line narrowing is slightly shifted toward higher $T$ as expected because of the larger rigid lattice line width. An additional shift by ca. $50 \mathrm{~K}$, if compared to the situation for LiNaTi $_{6} \mathrm{O}_{13}$, most likely also reflects a somewhat slower Li self-diffusion process in $\mathrm{Li}_{2} \mathrm{Ti}_{6} \mathrm{O}_{13}$. For $\mathrm{Li}_{0.33} \mathrm{Na}_{1.67} \mathrm{Ti}_{6} \mathrm{O}_{13}$ motional narrowing remains incomplete until ca. $500 \mathrm{~K}$. The difference in line widths is only $300 \mathrm{~Hz}$. Such a small difference is known from hydrogen diffusion in hydrides, where initial local motion or rotation leads only to a small reduction, while at higher $T$ line narrowing continues due to several jumps over longer distances [41, 42]. As lithium is diluted in $\mathrm{Li}_{0.33} \mathrm{Na}_{1.67} \mathrm{Ti}_{6} \mathrm{O}_{13}$ the partial narrowing seen might be attributed to local rather than long-range motion; Li mobility in Na-rich samples seems to be restricted because of the larger sodium ions.

Activation energies were estimated from a fit of the motional narrowing data using the empirical expression by Hendrickson and Bray [71]. The activation energies varied by about $0.1 \mathrm{eV}$ for the different $\mathrm{Li} / \mathrm{Na}$ ratios and range from 0.7 to $0.9 \mathrm{eV}$. A similar trend was observed by ${ }^{7} \mathrm{Li}$ NMR SLR.

In general, the $\mathrm{Li}_{x} \mathrm{Na}_{2-x} \mathrm{Ti}_{6} \mathrm{O}_{13}$ revealed relatively low rigid-lattice line widths. This fact and the observation of partial line narrowing for diluted $\mathrm{Li}_{0.33} \mathrm{Na}_{1.67} \mathrm{Ti}_{6} \mathrm{O}_{13}$ shows that the Li ions interact with only few neighbored spin-carrying ions as it would be the case inside a channel. Hence, most likely Li and Na diffusion is indeed restricted to the space inside the channels of the titanate.

\section{4 $\mathrm{LiBi}_{3} \mathrm{~S}_{5}$}

$\mathrm{LiBi}_{3} \mathrm{~S}_{5}$ also crystallizes with a channel-like structure and the Li ions residing inside the channels have, thus, access to a spatially rather restricted diffusion pathway (see Figure 11b). We followed a solid-state route to prepare phasepure gray-black $\mathrm{LiBi}_{3} \mathrm{~S}_{5}$ powder starting from $\mathrm{LiBiS}_{2}$ and $\mathrm{Bi}_{2} \mathrm{~S}_{3}$ [34]. Neutron diffractograms and lithium NMR spectra reveal its crystal structure to be a cation-disordered variety of the $\mathrm{AgBi}_{3} \mathrm{~S}_{5}$ type (synthetic pavonite; monoclinic, $C 2 / m)$. 

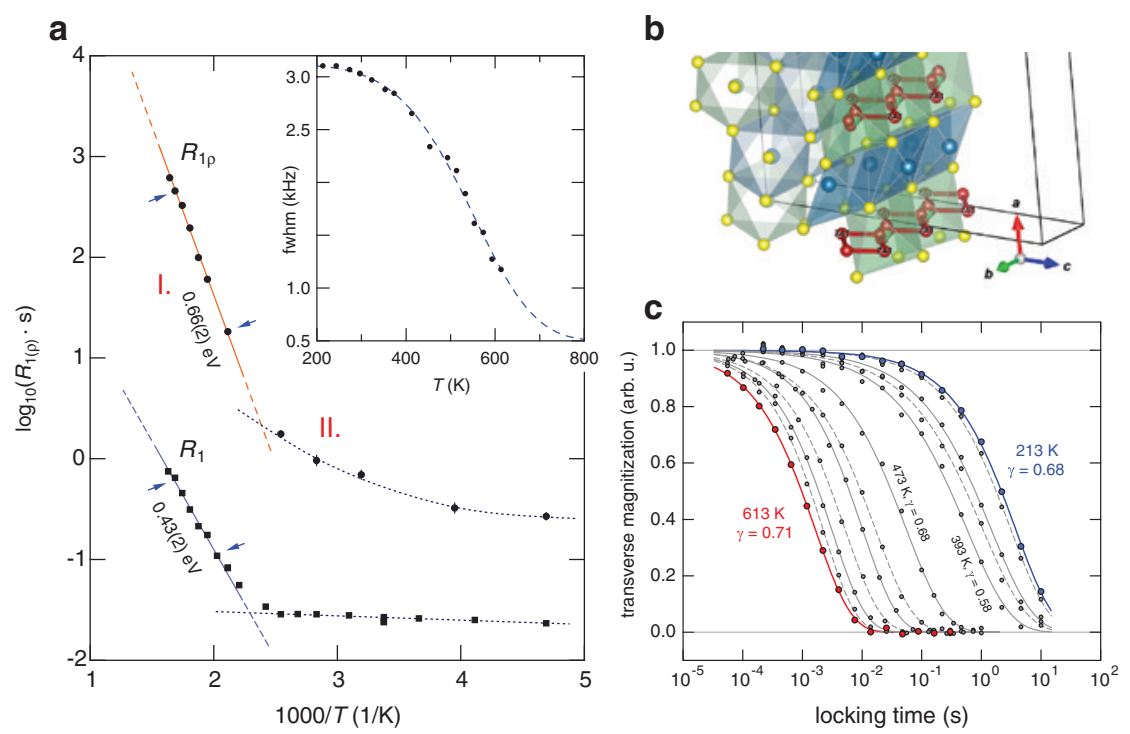

Fig. 11: (a) ${ }^{7} \mathrm{Li}$ SLR NMR rates $\left(1 / T_{1(\rho)} \equiv R_{1(\rho)}\right)$ of channel-structured $\mathrm{LiBi}_{3} \mathrm{~S}_{5}$ that offers $1 \mathrm{D}$ diffusion; the rates were recorded at $116 \mathrm{MHz}$ and $\omega_{1} / 2 \pi=20 \mathrm{kHz}$, respectively. Inset: ${ }^{~} \mathrm{Li}$ NMR line narrowing which refers to averaging of dipole-dipole interactions because of $\mathrm{Li}$ exchange with jump rates in the order of some kHz. (b) Illustration of the Li migration pathway, see Ref. [34] for further details. (c) Spin-lock magnetization transients from which the $1 / T_{1 p}$ rates shown in (a) were deduced.

Topological analyses and ${ }^{\mathrm{Li}} \mathrm{NMR}$ relaxometry suggest that the lithium ions indeed diffuse along the channels in $\mathrm{b}$ direction involving tetrahedral voids. Figure 11 shows the temperature dependence of the ${ }^{7} \mathrm{Li}$ NMR $1 / T_{1}$ and $1 / T_{1 \mathrm{p}}$ rates of $\mathrm{LiBi}_{3} \mathrm{~S}_{5}$. In the case of $1 / T_{1}$ above $340 \mathrm{~K}$ the diffusion-induced flank of the corresponding rate peak shows up; analyzing the flank yields an activation energy of $0.43 \mathrm{eV}$. At low temperatures the rates depend only weakly on $T$. The same features are essentially seen for $1 / T_{1 p}$. Above ambient temperature the rates already increase most likely because of some localized motional processes (regime II.). At higher $T$ we observed the flank of the $1 / T_{1 p}(1 / T)$ peak that yields $0.66 \mathrm{eV}$ (regime I.). Although the rate $1 / T_{1 \mathrm{p}}$ runs through different motional regimes the shape of the underlying transversal transients (see Figure 11c) does not change much. The transients can be best parametrized with stretched exponentials; the stretching factor $\gamma$ takes values from approximately 0.6 to 0.7 . The lowest values are found for the transition regime, i.e. when going from regime II. to I., see Figure 11a. The difference in activation energies $(0.43 \mathrm{eV}$ vs. $0.66 \mathrm{eV})$ can be explained by correlation effects. Obviously, compared to $1 / T_{1 p}$, they have a larger impact on the $1 / T_{1}$ rates 
being more sensitive to short-range Li ion diffusion rather than to long-range ion transport. For spatially confined ion migration such as 1D diffusion we indeed expect correlated motion and rather large activation energies.

The result obtained by NMR relaxometry is in agreement with the motional narrowing curve shown in the inset of Figure 11a. Although the rigid-lattice line width is rather low, viz. only slightly larger than $3 \mathrm{kHz}$, motional averaging of dipolar interactions extends over a rather wide temperature range. Even at $600 \mathrm{~K}$ the NMR line width has not reached the extreme narrowing regime. Thus, there is no doubt that $\mathrm{Li}$ ion diffusion in mixed-conducting $\mathrm{LiBi}_{3} \mathrm{~S}_{5}$ is rather poor. From a structural point of view we can explain this finding because of cation disorder. Our structural analyses [34] has shown that immobile $\mathrm{Bi}^{3+}$ ions may easily block the Li diffusion pathways, thus hindering ion migration along the channels of the sulfide. In such a case, Li ion diffusion is expected to be largely mediated by defects in the various sub-lattices enabling the ions to diffuse via non-regular lattice sites.

\subsection{Ramsdellite-type $\mathrm{Li}_{2} \mathrm{Ti}_{3} \mathrm{O}_{7}$}

Contrary to monoclinic $\mathrm{Li}_{2} \mathrm{Ti}_{3} \mathrm{O}_{7}$ having a layered structure, which we also examined with respect to low-dimensional Li diffusion [13], orthorhombic ramsdellite-type $\mathrm{Li}_{2} \mathrm{Ti}_{3} \mathrm{O}_{7}$ is a metastable titanate with relatively wide channels providing several inequivalent sites for the $\mathrm{Li}$ ions. Inside the channels the ions have access to various sites offering short-range and long-range ion diffusion. Whether Li ions do also occupy sites in the titanate framework is still under debate [36, 37, 75].

Our preliminary spin-lock ${ }^{7} \mathrm{Li}$ NMR SLR measurements are shown in Figure 12a. Above $200 \mathrm{~K}$ the diffusion-induced rate peaks show up. Although the peaks were measured at three different locking frequencies, we do not find significant shifts of the peak position with increasing spin-lock frequency. In contrast to the classical expectation for 1D diffusion, a significant frequency dependence in the high- $T$ limit is absent. Moreover, also in the limit $\omega_{0} \tau \gg 1$ only a shallow frequency dependence of the rate on locking frequency is seen. The solid line represents a fit taking into account the limiting behavior for channel-like diffusion, analyzing the rate peaks yields an activation energy of 0.43 $\mathrm{eV}$. The arrows drawn in Figure 12a indicate the positions of the peaks on the inverse temperature scale.

The slight frequency dependence of $1 / T_{1 \rho}$ in the limit $\omega_{0} \tau \gg 1$ might be attributed to the influence of strong local fields at the Li sites. Field-cycling (FC) NMR data carried out at $293 \mathrm{~K}$ [76] confirmed the results from $1 / T_{1 p}$ relaxometry; from $10^{3} \mathrm{~Hz}$ to $5 \times 10^{4} \mathrm{~Hz}$ the SLR rates $1 / \mathrm{T}_{1}$ are almost constant (see 
a
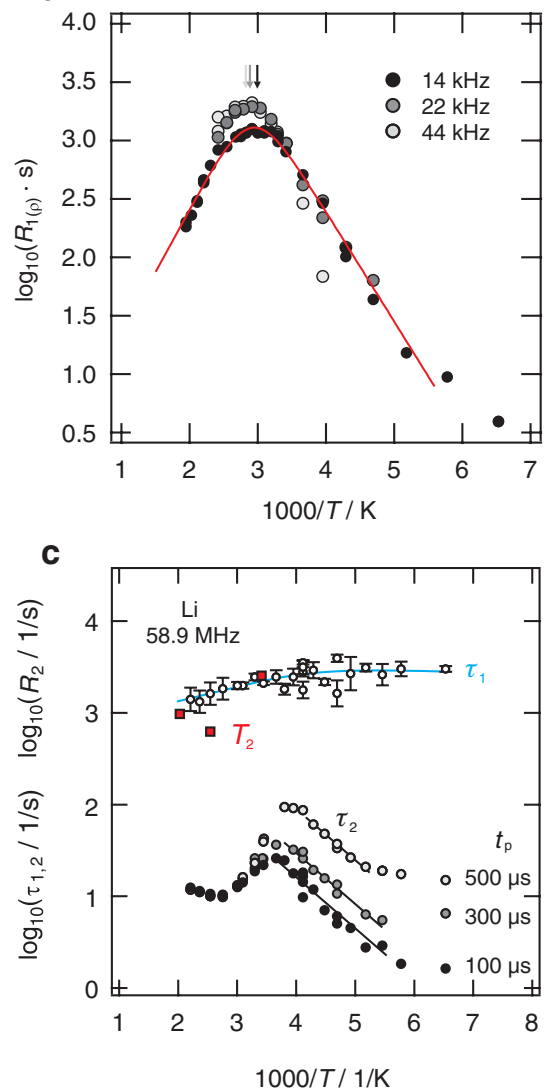

b

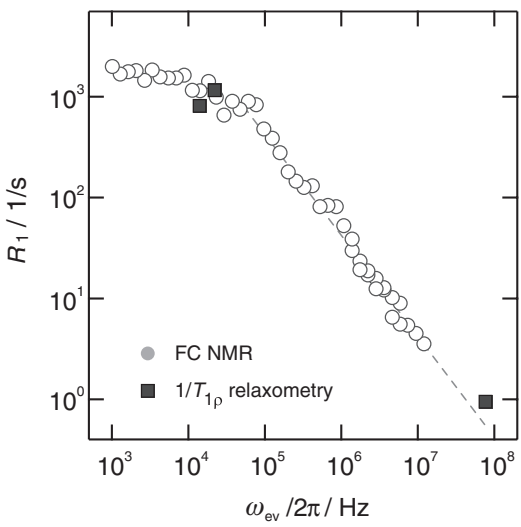

d

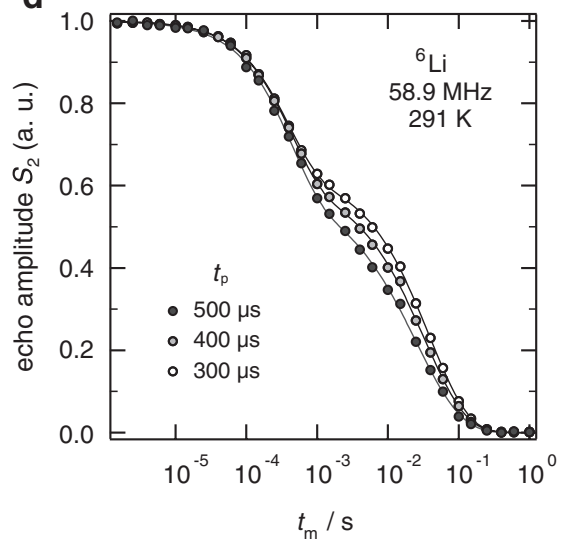

Fig. 12: (a) ${ }^{7} \mathrm{Li} N M R$ SLR rates of orthorhombic $\mathrm{Li}_{2} \mathrm{Ti}_{3} \mathrm{O}_{7}$ recorded in the rotating frame of reference at the locking frequencies $\omega_{1}$ indicated. The $1 / T_{1 p}$ rates recorded at $14 \mathrm{kHz}$ are analyzed using a model function for 1D diffusion. (b) Field-cycling NMR SLR rates (gray circles) recorded at an evolution frequency of $\omega_{\text {ev }} / 2 \pi=9.8 \mathrm{MHz}$ at $293 \mathrm{~K}$ over a frequency range of $1-10 \mathrm{MHz} .1 / T_{1 p}$ relaxometry data is shown for comparison (squares) and agrees well with the results from $\mathrm{FC}$ NMR. (c) ${ }^{6}$ Li SAE NMR data reveals two processes characterized by $1 / \tau_{1}$ and $1 / \tau_{2}$; the corresponding decay curves are shown in (d) for different preparation times $t_{\mathrm{p}}$. Solid lines in (d) represent stretched exponentials. Most likely, the first decay process is governed by spin-spin relaxation; $1 / T_{2}$ rates [see the squares in (c)] measured independently by the quadrupole-echo method are shown for comparison. The second decay step clearly depends on $t_{\mathrm{p}}$; the corresponding rates $1 / \tau_{2}$ are also included in (c). See text for further explanation.

Figure $12 \mathrm{~b}$ ). The rate $1 / T_{1}$ starts to depend on frequency for values above 100 $\mathrm{kHz}$. The diffusion process seen via both FC NMR and spin-locking NMR might represent local Li ion exchange within the channels; this dynamic 
process would be in line with the structural model proposed by Orera et al. [75] based on Li MAS NMR assuming the Li ions to only occupy intrachannel sites in $\mathrm{Li}_{2} \mathrm{Ti}_{3} \mathrm{O}_{7}$. This assumption is corroborated by a recent high-temperature neutron diffraction study showing that the Li ions exclusively occupy interstitial, i.e. intrachannel, positions in ramsdellite-like $\mathrm{Li}_{2} \mathrm{Ti}_{3} \mathrm{O}_{7}$ rather than positions in the titanate framework [36].

To further elucidate the dynamic processes in $\mathrm{Li}_{2} \mathrm{Ti}_{3} \mathrm{O}_{7},{ }^{6} \mathrm{Li}$ SAE NMR measurements $[7,64,77]$ were carried out as a function of both mixing time $\left(t_{\mathrm{m}}\right)$ and preparation time, $t_{\mathrm{p}}$ (see Figure $12 \mathrm{c}$ and $\mathrm{d}$ ). The pulse sequence introduced by Jeener and Broeckaert [78] was used to measure the two-time single-spin correlation functions $S_{2}$. The curves $S_{2}\left(t_{\mathrm{p}}=\right.$ const., $\left.t_{\mathrm{m}}\right)$, obtained by plotting the echo intensity of the stimulated echo as a function of $t_{\mathrm{m}}$, reveal a two-step decay (Figure 12d). A sum of two stretched exponentials was used to analyze the data. Presumably, the first decay step, which is characterized by the rate constant $1 / \tau_{1}$ represents quadrupolar spin-spin relaxation and agrees well with the independently measured spin-spin-relaxation rate $T_{2}$, which is controlled by both dipolar as well as quadrupolar fluctuations, see Figure 12c. The second decay step is characterized by $\tau_{2} \cdot 1 / \tau_{2}$ starts to be thermally activated at temperatures higher than $200 \mathrm{~K}$. The solid lines in Figure 12c represent fits with an Arrhenius relation yielding approx. $0.1 \mathrm{eV}$. This value is independent of the preparation time used to acquire the stimulated echoes. In general, with $t_{\mathrm{p}}$ the sensitivity of the experiment is set with regard to electricquadrupolar fluctuations sensed by the Li ions. Thus, one would expect the decay rate $1 / \tau_{2}$ to increase with increasing $t_{\mathrm{p}}$. Moreover, the increasing generation of dipolar contributions to the spin-alignment echo at enhanced $t_{\mathrm{p}}$ might lead to an additional increase of $1 / \tau_{2}$. At sufficiently high $T$, quadrupolar interactions are averaged because of rapid Li exchange between the positions involved. As a consequence, stimulated echoes are no longer able to distinguish between the electrically inequivalent Li sites, and the second decay step of the $S_{2}$ curves shift toward longer mixing times. Hence, the rate $1 / \tau_{2}$ passes through a maximum. For $t_{\mathrm{p}}=100 \mu$ s the rates $1 / \tau_{2}$ superimpose a non-diffusive background contribution. At high temperatures the $S_{2}$ decay seems to be influenced by spin-diffusion usually characterized by temperature independent rates with values in the order of $1 \mathrm{~s}^{-1}$.

The effective averaging of electric quadrupolar interactions at temperatures as low as $250 \mathrm{~K}$ in combination with rather low activation energies suggests that ${ }^{6} \mathrm{Li}$ SAE NMR senses local Li jumps between the close-by sites within the channels. Assuming electric field gradients with coupling constants in the order of $10^{4} \mathrm{~Hz}$, between $285 \mathrm{~K}$ and $250 \mathrm{~K}$ the Li residence times should be in the order of the inverse coupling constants of $0.1 \mathrm{~ms}$. 


\section{Summary}

Time-domain solid-state nuclear magnetic resonance is a flexible tool to access dynamic parameters of mobile ions in crystalline as well as amorphous materials. The combination of relaxation NMR with field-gradient techniques, stimulated echo NMR or even field cycling NMR allows the investigation of translational ion dynamics over a wide dynamic range, covering in the best case several orders of magnitude. Here, we used selected model substances with quite different structural features to analyze $\mathrm{Li}$ and $\mathrm{Na}$ ion dynamic parameters to understand the interplay of both short-range and long-range hopping processes in crystalline solids. The compounds were chosen such that they proffer migration pathways with reduced dimensionality, thus offering layered structures $\left(\mathrm{LiBH}_{4}, \mathrm{LiC}_{6}\right.$, $\mathrm{Li}_{x} \mathrm{MeS}_{2}(\mathrm{Me}=\mathrm{Ti}, \mathrm{Nb}, \mathrm{Sn} ; 0<x \leq 1)$ or channels $\left(\mathrm{LiBi}_{3} \mathrm{~S}_{5}, \mathrm{Li}_{2} \mathrm{Ti}_{3} \mathrm{O}_{\rightarrow} \mathrm{Li}_{x} \mathrm{Nb}_{3} \mathrm{~S}_{4}, \mathrm{Li}_{12} \mathrm{Si}_{7}\right)$ to guide the ions.

In ideal cases, NMR relaxometry, regardless of being carried out in the laboratory or rotating frame of reference, is able to sense the dimensionality of the diffusion process. There is no need for single crystals. Analyzing the frequency dependence of the NMR spin-lattice relaxation rates serves as the ultimate tool of NMR spin-lattice relaxation to judge whether a diffusion process is 3D, 2D or $1 \mathrm{D}$ of nature. This analysis is only possible if the so-called high-temperature flank of the diffusion-induced rate peak is experimentally accessible. For instance, via frequency-dependent NMR SLR measurements we revealed 2D Li ion diffusion in the hexagonal modification of $\mathrm{LiBH}_{4}$ as well as the transition metal dichalcogenides, see the $2 \mathrm{H}$ and $3 \mathrm{R}$ form of $\mathrm{Li}_{x} \mathrm{NbS}_{2}$. In particular, for lithium boron hydride we clearly showed the logarithmic frequency dependence of the relaxation rate $1 / T_{1}$, which is in excellent agreement with the spin-lattice relaxation model for $2 \mathrm{D}$ diffusion proposed by Richards. While intrachannel diffusion in $\mathrm{LiBi}_{3} \mathrm{~S}_{5}$ and $\mathrm{Li}_{x} \mathrm{Na}_{2-x}-\mathrm{Ti}_{6} \mathrm{O}_{13}$ turned out to be rather slow, most likely because of blocking effects, the wider channels in $\mathrm{Li}_{2} \mathrm{Ti}_{3} \mathrm{O}_{7}$ seem to ensure fast $\mathrm{Li}$ exchange. Fast quasi-1D diffusion has also been used to explain the occurrence of $1 / T_{1 p}$ rate peaks in the Zintl phase $\mathrm{Li}_{12} \mathrm{Si}_{\vec{p}}$, which showed up at unexpectedly low temperatures. Both the asymmetry of the peaks and the frequency dependence in the high- $T$ limit suggest spatially restricted Li motions. Obviously, the stacked $\mathrm{Si}_{5}$ rings guide the ions along a channel-like diffusion pathway.

Currently, also other 2D and 1D ion conductors are being studied in our laboratories to evaluate relaxation models introduced to describe low-dimensional ion transport. The search for systems which clearly show the signatures of quasi $1 \mathrm{D}$ diffusion is particularly challenging. Answering the question how and to what extent dimensionality is able to influence ion dynamics is expected to advance our efforts in developing new functional materials with controllable transport parameters besides being a fundamental issue. 
Acknowledgement: We appreciate financial support by the Deutsche Forschungsgemeinschaft, grants no. DFG FOR 1277 molife HE1574/11-1, 11-2, WI3600/2-1, 2-2, and LE781/15-2. We are grateful to Prof. F. Fujara and his group at TU Darmstadt for help with the FC NMR measurements and the permission to show Fig. 12b prior to publication (cf. Ref. [76]).

\section{References}

1. D. F. Holcomb, R. E. Norberg, Phys. Rev. 98 (1955) 1074.

2. H. S. Gutowsky, B. R. McGarvey, J. Chem. Phys. 20 (1952) 1472.

3. R. Böhmer, K. R. Jeffrey, M. Vogel, Prog. Nucl. Mag. Res. Sp. 50 (2007) 87.

4. C. V. Chandran, P. Heitjans, Ann. Rep. NMR Spectrosc. 89 (2016) 1.

5. P. Heitjans, A. Schirmer, S. Indris, in: P. Heitjans, J. Kärger (Eds.): Diffusion in Condensed Matter: Methods, Materials, Models, Springer, Berlin and New York (2005), P. 367.

6. A. Kuhn, S. Narayanan, L. Spencer, G. Goward, V. Thangadurai, M. Wilkening, Phys. Rev. B 83 (2011) 094302.

7. M. Wilkening, P. Heitjans, Chem. Phys. Chem. 13 (2012) 53.

8. V. Epp, M. Wilkening, in: N. J. Dudney, W. C. West, J. Nanda (Eds.): Handbook of Solid State Batteries, World Scientific Publishing, New Jersey, Hong Kong (2015), P. 133.

9. M. Uitz, V. Epp, M. Wilkening, J. Electroceram. in press (2017). doi: 10.1007/s10832-0170071-4.

10. A. Kuhn, M. Kunze, P. Sreeraj, H. D. Wiemhöfer, V. Thangadurai, M. Wilkening, P. Heitjans, Solid State Nucl. Mag. 42 (2012) 2.

11. W. Küchler, P. Heitjans, A. Payer, R. Schöllhorn, Solid State Ion. 70-71 (1994) 434.

12. V. Epp, M. Wilkening, Phys. Rev. B. 82 (2010) 020301.

13. K. Volgmann, V. Werth, S. Nakhal, M. Lerch, T. Bredow, P. Heitjans, Z. Phys. Chem. 231 (2017) 1243.

14. N. Bloembergen, E. M. Purcell, R. V. Pound, Phys. Rev. 73 (1948) 679.

15. A. Bunde, W. Dieterich, P. Maas, M. Meyer, in: P. Heitjans, J. Kärger (Eds.): Diffusion in Condensed Matter: Methods, Materials, Models, Springer, Berlin and New York (2005), P. 813.

16. P. M. Richards, in: M. B. Salamon (Ed.): Physics of Superionic Conductors, Springer, Berlin Heidelberg (1979), P. 141.

17. C. A. Sholl, J. Phys. C Solid State Phys. 14 (1981) 447.

18. A. F. McDowell, C. F. Mendelsohn, M. S. Conradi, R. C. Bowman, A. J. Maeland, Phys. Rev. B. 51 (1995) 6336.

19. F. Fujara, D. Kruk, A. F. Privalov, Prog. Nucl. Mag. Res. Sp. 82 (2014) 39.

20. U. Alpen, H. Schulz, G. H. Talat, H. Böhm, Solid State Commun. 23 (1977) 911.

21. D. P. Almond, A. R. West, R. J. Grant, Solid State Commun. 44 (1982) 1277.

22. D.L. Sidebottom, Phys. Rev. Lett. 83 (1999) 983.

23. M. Villa, J. L. Bjorkstam, G. Mariotto, A. Fontana, E. Cazzanelli, J. Chem. Phys. 76 (1982) 2804.

24. M. T. Chowdhury, R. Takekawa, Y. Iwai, N. Kuwata, J. Kawamura, J. Chem. Phys. 140 (2014) 124509.

25. M. S. Whittingham, J. Solid State Chem. 12 (1978) 41. 
26. M. Wilkening, W. Küchler, P. Heitjans, Phys. Rev. Lett. 97 (2006) 65901.

27. M. Wilkening, P. Heitjans, Phys. Rev. B. 77 (2008) 24311.

28. V. Epp, M. Wilkening, Chem. Phys. Chem. 14 (2013) 3706.

29. V. Epp, S. Nakhal, M. Lerch, M. Wilkening, J. Phys. Condens. Matter. 25 (2013) 195402.

30. B. Stanje, V. Epp, S. Nakhal, M. Lerch, M. Wilkening, ACS Appl. Mater. Interfaces. 7 (2015) 4089.

31. J. Langer, V. Epp, M. Sternad, M. Wilkening, Solid State Ion. 276 (2015) 56.

32. J. Langer, V. Epp, P. Heitjans, F. A. Mautner, M. Wilkening, Phys. Rev. B. 88 (2013) 094304.

33. K. Volgmann, A. Schulz, A.-M. Welsch, T. Bredow, S. Nakhal, M. Lerch, P. Heitjans, Z. Phys. Chem. 229 (2015) 1351.

34. S. Nakhal, D. Wiedemann, B. Stanje, O. Dolotko, M. Wilkening, M. Lerch, J. Solid State Chem. 238 (2016) 60.

35. J. Heine, M. Wilkening, P. Heitjans, Diffus. Fundam. 11 (2009) 47.

36. D. Wiedemann, S. Nakhal, A. Franz, M. Lerch, Solid State Ion. 293 (2016) 37.

37. M. M. Islam, P. Heitjans, T. Bredow, J. Phys. Chem. C. 120 (2016) 5.

38. A. Kuhn, P. Sreeraj, R. Pöttgen, H. D. Wiemhöfer, M. Wilkening, P. Heitjans, Angew. Chem. Int. Edit. 50 (2011) 12099.

39. A. Kuhn, S. Dupke, M. Kunze, S. Puravankara, T. Langer, R. Pöttgen, M. Winter, H. D. Wiemhöfer, H. Eckert, P. Heitjans, J. Phys. Chem. C. 118 (2014) 28350.

40. A. Kuhn, P. Sreeraj, R. Pöttgen, H.-D. Wiemhöfer, M. Wilkening, P. Heitjans, J. Am. Chem. Soc. 133 (2011) 11018.

41. A. V. Soloninin, A. V. Skripov, A. L. Buzlukov, A. P. Stepanov, J. Solid State Chem. 182 (2009) 2357.

42. A. V. Skripov, A. V. Soloninin, L. H. Rude, T. R. Jensen, Y. Filinchuk, J. Phys. Chem. C. 116 (2012) 26177.

43. M. Matsuo, Y. Nakamori, S. I. Orimo, H. Maekawa, H. Takamura, Appl. Phys. Lett. 91 (2007) 224103.

44. P. C. Aeberhard, K. Refson, W. I. David, Phys. Chem. Chem. Phys. 15 (2013) 8081.

45. M. Matsuo, H. Takamura, H. Maekawa, H. W. Li, S. I. Orimo, Appl. Phys. Lett. 94 (2009) 084103.

46. H. Maekawa, M. Matsuo, H. Takamura, M. Ando, Y. Noda, T. Karahashi, S. I. Orimo, J. Am. Chem. Soc. 131 (2009) 894.

47. P. M. Richards, Solid State Commun. 25 (1978) 1019.

48. P. C. Aeberhard, S. R. Williams, D. J. Evans, K. Refson, W. I. David, Phys. Rev. Lett. 108 (2012) 95901.

49. J. S. Myrdal, D. Blanchard, D. Sveinbjörnsson, T. Vegge, J. Phys. Chem. C. 117 (2013) 9084.

50. K. Funke, Prog. Solid State Chem. 22 (1993) 111.

51. T. Pietrass, F. Taulelle, P. Lavela, J. Olivier-Fourcade, J.-C. Jumas, S. Steuernagel, J. Phys. Chem. B. 101 (1997) 6715.

52. J. Morales, C. Perezvicente, J. Tirado, Solid State Ion. 51 (1992) 133.

53. A. Herold, Synthetic Met. 23 (1988) 27.

54. P. Freiländer, P. Heitjans, A. Ackermann, B. Bader, G. Kiese, A. Schirmer, H.-J. Stöckmann, C. van der Marel, A. Magerl, H. Zabel, Z. Phys. Chem. N. F. 151 (1987) 93.

55. A. Schirmer, P. Heitjans, Z. Naturforsch. 50a (1995) 643.

56. A. Magerl, H. Zabel, I. S. Anderson, Phys. Rev. Lett. 55 (1985) 222.

57. K. Toyoura, Y. Koyama, A. Kuwabara, F. Oba, I. Tanaka, Phys. Rev. B. 78 (2008) 214303.

58. S. Thinius, M. M. Islam, P. Heitjans, T. Bredow, J. Phys. Chem. C. 118 (2014) 2273. 
59. K. Boesebeck, C. V. Chandran, B. K. Licht, M. Binnewies, P. Heitjans, Energy Technol. 4 (2016) 1598.

60. D. Burow, K. Sergeeva, S. Calles, K. Schorb, A. Börger, C. Roth, P. Heitjans, J. Power Sources. 307 (2016) 806.

61. J. Shi, Z. Wang, Y. Q. Fu, Electrochim. Acta. 186 (2015) 71.

62. A. F. J. Ruysink, F. Kadijk, A. J. Wagner, F. Jellinek, Acta Cryst. B. 24 (1969) 1614.

63. Y. G. Kriger, A. V. Mishchenko, A. R. Semenov, S. V. Tkachev, V. E. Fedorov, Phys. Solid State +. 42 (2000) 257.

64. M. Wilkening, D. Gebauer, P. Heitjans, J. Phys. Condens. Matter. 20 (2008) 022201.

65. A. Kuhn, P. Heitjans, Diffus. Fundam. 12 (2010) 97.

66. M. Wilkening, R. Amade, W. Iwaniak, P. Heitjans, Phys. Chem. Chem. Phys. 9 (2007) 1239.

67. W. Iwaniak, J. Fritzsche, M. Zukalová, R. Winter, M. Wilkening, P. Heitjans, Defect Diffus. Forum 289-292 (2009) 565.

68. M. Wilkening, C. Mühle, M. Jansen, P. Heitjans, J. Phys. Chem. B 111 (2007) 8691.

69. S. Andersson, A. D. Wadsley, Acta Cryst. 15 (1962) 194.

70. K. Kataoka, J. Awaka, N. Kijima, H. Hayakawa, K.-I. Ohshima, J. Akimoto, Chem. Mater. 23 (2011) 2344.

71. J. Hendrickson, P. Bray, J. Magn. Res. 9 (1973) 341.

72. A. M. Gil, E. Alberti, Solid State Nucl. Mag. 11 (1998) 203.

73. J. van Kranendonk, Physica. 20 (1954) 781.

74. J. H. Van Vleck, TPhys. Rev. 74 (1948) 1168.

75. A. Orera, M. T. Azcondo, F. García-Alvarado, J. Sanz, I. Sobrados, J. Rodríguez-Carvajal, U. Amador, Inorg. Chem. 48 (2009) 7659.

76. K. Volgmann, B. Kresse, A. F. Privalov, F. Fujara, P. Heitjans, 7Li Field-cycling NMR as Powerful Tool for Investigating Li lon Conductors, in: P. Heitjans (Ed.): Bunsen Colloquium 'Lithium in Solids: Structure and Dynamics': Book of Abstracts, 2014, P. 33 . Also online: diffusionfundamentals.org 21 (2014) 25.

77. M. Wilkening, A. Kuhn, P. Heitjans, Phys. Rev. B 78 (2008) 054303.

78. J. Jeener, P. Broekaert, Phys. Rev. 157 (1967) 232. 